\title{
Timing Idiosyncratic Volatility and Dynamic Asset Allocation
}

\author{
Yun SHI \\ Academy of Statistics and Interdisciplinary Sciences \\ Faculty of Economics and Management \\ East China Normal University
}

\begin{abstract}
We study the multi-period portfolio selection problem of a mean-variance investor that allocates his wealth across assets in an environment where the expected return, the idiosyncratic volatility, and the corresponding transaction cost are time-varying. Specifically, we assume that asset return and idiosyncratic volatility are predicted by a factor structure, and that transaction cost is of quadratic form. Our feedback trading strategy indicates that the investor should trade partially from the current position toward a dynamic aim portfolio, which is a weighted average of the terms related to the future Markowitz's tangency portfolio. Our analysis offers new insights to the existing literature. First, our optimal trading strategy is highly affected by the common factor of idiosyncratic volatility (CIV), which is new in literature. CIV turns out to be a reliable signal that alarm both deteriorating investment opportunities and high level of transaction cost. Secondly, the investor will trade more closely toward the aim portfolio with a more stable risk signals. The empirical out-of-sample tests further demonstrate that our dynamic trading strategy outperforms two benchmark strategies and the benefit mainly arises from timing idiosyncratic volatility.

Key words: Idiosyncratic volatility, dynamic asset allocation, transaction cost, return predictability, volatility timing.
\end{abstract}




\section{Introduction}

Dynamic asset allocation problem provides active investors, e.g., hedge funds, a framework for determining and evaluating optimal investment strategies. This central and essential problem has a long history dating back to Mossin (1968), Samuelson (1969), and Merton (1969). However, due to the non-separable structure of variance term, the optimal strategies under dynamic mean-variance framework are obtained till 2000 (see Li and Ng, 2000, Zhou and Li, 2000), and the optimal dynamic strategies with different constraints are further studied, such as Li et al. (2002), Gao and Li (2013), Cui et al. (2014) etc. In practice, active investors often rely on different return predictors, say, value or momentum factors, to predict asset returns and trade accordingly to profit from their predictions. To best exploit the return predictability, the investor should also take risk prediction into consideration as both return and risk characterize the whole picture of how investment opportunity evolves along time. Meanwhile, transaction cost is also important in the case of dynamic trading as the optimal strategies that design to profit from various predictions often rebalance frequently, resulting in large transaction cost.

Although it seems quite reasonably to consider all three elements: return and risk predictability and transaction cost, in asset allocation practice, few studies in academic literature studying the optimal trading strategies in presence of all those three elements. This is because introducing transaction cost in a standard dynamic portfolio choice problem tends to make it intractable. ${ }^{1}$ Indeed, most academic papers studying transaction cost focus on limited predictability, and often rely on numerical solutions.

\footnotetext{
${ }^{1}$ Optimal portfolio selection problem with transaction cost was first studied by Constantinides (1986), which models transaction cost as proportional bid-ask spread. Liu (2004) obtained a closed-form solution to
} 
Optimal portfolio selection problem which combining return predictability and transaction cost was first studied by Balduzzi and Lynch (1999). By incorporating proportional and fixed transaction cost, Balduzzi and Lynch (1999) showed that substantial loss in utility would occur when the agent ignores return predictability and transaction cost. Lynch and Balduzzi (2000) examined the impact of return predictability and transaction cost on the agent's rebalancing behavior. Under quadratic form transaction cost, Gârleanu and Pedersen (2013) (GP model) obtained an explicit solution to the portfolio selection problem of an infinitely-lived mean-variance investor with return predicting signals in a discretetime framework. Gârleanu and Pedersen (2016) further extended their investigations to a continuous-time framework. ${ }^{2}$ In this paper, we further extend GP model to incorporate the risk predictability by considering a factor structure in idiosyncratic volatility. Our model wants to provide a highly tractable framework for studying the effects of all three elements, the risk and return predictability and transaction cost, on portfolio performance.

Idiosyncratic volatility represents the uncertainty in returns that cannot be explained by systematic risk factors (see Ang et al., 2006). Traditional portfolio selection theories normally ignore idiosyncratic volatility, under the assumption that holding idiosyncratic risk (diversifiable risk) should not be rewarded. However, recent studies (for example, Duarte et al., 2014 and Herskovic et al., 2016) identify a robust common factor in idiosyncratic volatility,

the optimal portfolio problem with multiple, mutually independent assets for the case of a constant absolute risk-averse (CARA) agent with both fixed and proportional costs.

${ }^{2}$ There are many other studies in recent literature extending the scope of Gârleanu and Pedersen (2013)'s analysis. For example, Glasserman and Xu (2013) extends to examine the robustness in model uncertainty. Zhang et al. (2017) further considers the impact of the ambiguity aversion on returns and return-predicting signals. Collin-Dufresne et al. (2019) considers a general regime-switch dynamics in expected returns. 
implying that a undiversifiable component exists in idiosyncratic volatility. Therefore, the investor should take this kind of undiversifiable risk into consideration when doing their investment. This paper incorporates this kind of risk predictability into a dynamic asset allocation problem by introducing a factor structure in idiosyncratic volatility.

This paper addresses how the optimal trading strategy depends on assets' return predictability, risk predictability, and transaction cost. We get a closed-form solution for the optimal dynamic trading strategies under a multi-period mean-variance framework with quadratic form transaction cost. The optimal trading strategy is to rebalance partially towards an aim portfolio, which is a weighted average of the expected Markowitz tangency portfolios for all future periods. The general trading rules that arising from transaction cost (as shown in GP's model) still preserve in our model. But, we do find several new implications driven by idiosyncratic volatility as indicated in our optimal trading strategies.

The key novelty in our model is modeling the evolution of idiosyncratic risk by introducing a factor structure in idiosyncratic volatility. Common fluctuations in idiosyncratic volatility (CIV), thus, enters the optimal trading strategy. By analyzing the derived optimal trading strategy, both theoretically and empirically, we first uncover the role of CIV as a risk alarm signal. Whenever there is a high level of CIV, the investor will scale down all his optimal positions in risky assets simultaneously. Secondly, high level of CIV also represents high level of transaction cost, the investor will trade less frequently to economize on transaction cost. Thirdly, we find that the more stable of CIV signal, the investor would rebalance much more toward the aim portfolio. In other words, the investor would trade more confidently to profit from their return information with a more stable risk signal.

Our paper also contributes to the volatility timing literature. Fleming et al. (2001) and 
Fleming et al. (2003) first investigated its economic value of volatility timing by constructing and testing volatility timing strategies empirically. Using mutual fund data, Busse (1999) found that fund managers indeed benefit a lot from volatility timing in practice. Moreira and Muir (2017) showed that various factors' Sharpe ratios can be improved by timing factors' volatility. Moreira and Muir (2018) claimed that the long-term investor who ignores timing volatility would suffers a lot. Our study provides further evidence on volatility timing from the new perspective of idiosyncratic volatility timing.

We further present two empirical applications of our framework in which: i) a fund manager decides to move in and out the stock market; ii) a fund manager decides asset allocation across five different industries. We compare the out-of-sample performance of our optimal dynamic strategy to two alternatives: a baseline strategy which does not consider any time variation both in expected return and idiosyncratic volatility; and the GP strategy which considers only time-varying expected returns, but ignores the dynamics of idiosyncratic volatility and transaction cost. We find that the net-of-cost performance of our dynamic trading strategy is higher than the other two alternative strategies and the benefits mainly come from timing time changes in idiosyncratic volatility and the corresponding transaction cost.

More specifically, we measure out-of-sample performance of each strategy by the realized terminal wealth, accumulated risk penalty, transaction cost and (net) Sharpe ratio. Since our model captures the time-variation in risk and the corresponding changes in transaction costs more accurately, our optimal strategy is able to manage the risk-exposure and the incurred transaction costs more reliably, which directly contributes to net performance of our strategy. Moreover, we find very large gains from idiosyncratic volatility timing, which we measured by 
the additional benefits of switching from the GP strategy (which only conditions on expected return) to our fully optimal strategy (which conditions on idiosyncratic volatility as well as expected returns). In terms of net Sharpe ratio, timing idiosyncratic volatility contributes around $88 \%$ (for market timing case) or $60 \%$ (for industry allocation case) of the total gain of switching from the baseline strategy (which does not do any timing) to our fully optimal strategy. Thus, ignoring variation in idiosyncratic volatility is very costly and the benefits of timing idiosyncratic volatility are larger than the benefits of timing expected returns.

The rest of this paper is organized as follows. In Section 2, factor structures in return and risk is presented. Section 3 lays out the optimal portfolio model, and provides the close-form optimal solution. Section 4 provides several implications of our optimal trading strategy theoretically. Section 5 bring our model to data to test its empirical performance. Section 6 concludes the paper. All proofs are in the Appendix.

\section{Factor Structures in Return and Idiosyncratic Volatility}

There are $N$ risky stocks and one risk-free bond in the market. The risk-free return is $r_{f}$. The excess returns of stocks over the risk-free return in period $t, \boldsymbol{r}_{t+1}$, follow a factor model,

$$
\boldsymbol{r}_{t+1}=\boldsymbol{B} \boldsymbol{f}_{r, t}+\boldsymbol{u}_{t+1},
$$

where $\boldsymbol{f}_{r, t}$ is a $K \times 1$ vector of factors that predict returns, $\boldsymbol{B}$ is a $N \times K$ matrix of factor loadings, $\boldsymbol{u}_{t+1}$ is the unpredictable zero-mean noise term with covariance matrix $\boldsymbol{\Sigma}_{t+1}$. As the factor $\boldsymbol{f}_{r, t}$ is known to the investor already at time $t$, the conditional covariance matrix of $\boldsymbol{r}_{t+1}$ equals to $E_{t}\left[\boldsymbol{\Sigma}_{t+1}\right]$. In other words, at time $t$, the uncertainty of $\boldsymbol{r}_{t+1}$ only comes from 
$\boldsymbol{u}_{t+1}$ (and more specifically, is measured by $\boldsymbol{\Sigma}_{t+1}$ ).

The return-predicting factor $\boldsymbol{f}_{r, t}$ evolves according to a mean-reverting process,

$$
\boldsymbol{f}_{r, t+1}=(\boldsymbol{I}-\boldsymbol{\Phi}) \boldsymbol{f}_{r, t}+\boldsymbol{\epsilon}_{t+1},
$$

where $\boldsymbol{\Phi}$ is a $K \times K$ matrix of mean-reversion coefficients for the factors, and $\boldsymbol{\epsilon}_{t+1}$ is the shock affecting the predictors with constant covariance matrix $\boldsymbol{\Sigma}^{\epsilon}$. $(\boldsymbol{I}-\boldsymbol{\Phi})$ represents the persistence of the return signal, which measures how long the investor can enjoy the highly predicted return. Till now, we basically repeat the model adopted by Gârleanu and Pedersen (2013), which characterises the return predictability in stocks. Next, we further extend GP's model to incorporate risk predictability by considering a factor structure in volatilities.

The return-predicting factor $\boldsymbol{f}_{r, t}$ can be any factors, including macroeconomic and microeconomic factors, etc. Take the Fama-French three factor model (FF3) for example, $\boldsymbol{f}_{r, t}=\left(M K T_{t}, H M L_{t}, S M B_{t}\right)^{T}$. The standard deviation of $u_{t+1}^{i}$, which is denoted as $\sigma_{t+1}^{i}$, is known as idiosyncratic volatility in the literature. Moreover, recent studies, such as Duarte et al. (2014) and Herskovic et al. (2016), have documented a strong common factor in idiosyncratic volatilities across different assets, which implies that the idiosyncratic volatilities of assets also have a factor structure. Furthermore, they have reported that the common factor of idiosyncratic volatility (CIV) is indeed the average of idiosyncratic volatilities of all stocks in the market, which is proxied by $f_{\sigma}$ in this paper.

Following Ding et al. (2020), we set the factor structure for idiosyncratic volatilities as follows,

$$
\sigma_{t+1}^{i}=f_{\sigma, t+1} e^{k_{i}+\ell_{i} Z_{i, t+1}}
$$


where $Z_{i, t+1}$ is a standard normal random variable, $Z_{i, t+1}$ and $f_{\sigma, t+1}$ are independent, and $Z_{i, t+1}$ and $Z_{j, t+1}$ are also independent for different $i$ and $j .{ }^{3}$ Note that, the factor model proposed is a particular linear model for $\ln \left(\sigma_{t+1}^{i}\right)$. Bekaert et al. (2012) found that the common factor of idiosyncratic variance follows a autoregressive (AR) process that occasionally switches into a higher-variance regime but with relatively short duration. To simplify the mathematical derivations, we assume that $f_{\sigma, t}^{2}$ follows an simple $\mathrm{AR}(1)$ model,

$$
f_{\sigma, t+1}^{2}=\psi f_{\sigma, t}^{2}+\zeta_{t+1}
$$

where $\zeta_{t+1}$ is the noise term with variance $\sigma_{\zeta}^{2}$, and $\psi$ measures the persistence of CIV signal. We can recover the whole variance-covariance structure of $\boldsymbol{u}_{t+1}$ by further assuming that the correlation coefficient matrix between the noise terms of different stocks is a constant matrix $\boldsymbol{\rho}=\left(\rho_{i j}\right)$.

According to the proposed factor structure, we can derive the following conditional expectations of the excess returns,

$$
\begin{aligned}
& E_{t}\left[\boldsymbol{r}_{t+1}\right]=\boldsymbol{B} \boldsymbol{f}_{r, t}, \\
& E_{s}\left[\boldsymbol{r}_{t+1}\right]=\boldsymbol{B}(\boldsymbol{I}-\boldsymbol{\Phi})^{t-s} \boldsymbol{f}_{r, s}, \quad s<t .
\end{aligned}
$$

We can also derive the following conditional covariance matrixes,

$$
\begin{aligned}
& E_{t}\left[\boldsymbol{\Sigma}_{t+1}\right]=\boldsymbol{\Omega} \psi f_{\sigma, t}^{2}, \\
& E_{s}\left[\boldsymbol{\Sigma}_{t+1}\right]=\boldsymbol{\Omega} \psi^{(t+1-s)} f_{\sigma, s}^{2}, \quad s<t,
\end{aligned}
$$

\footnotetext{
${ }^{3}$ Herskovic et al. (2016) proposed that the idiosyncratic volatility linearly relates to CIV. However, Ding et al. (2020) argued that linear model is not suitable by documenting that the square of the residual in linear regression has strongly positive correlation with CIV.
} 
with the elements of the constant matrix given as follows,

$$
\begin{aligned}
& \boldsymbol{\Omega}(i j)=\rho_{i j} e^{k_{i}+\ell_{i}^{2} / 2} e^{k_{j}+\ell_{j}^{2} / 2}, \quad i \neq j, \\
& \boldsymbol{\Omega}(i i)=e^{2 k_{i}+2 \ell_{i}^{2}} .
\end{aligned}
$$

Before moving into a formal dynamic portfolio selection model, we need to understand the proposed factor structure in a dynamic trading environment first. Consider an active investor, for example, an asset manager, who uses different return predictors (collected in vector $\boldsymbol{f}_{r, t}$ ), to predict securities returns and tries to trade accordingly to profit from their predictions. Such dynamic trading often involves large turnover and transaction costs. Therefore, the active manager should constantly balance the expected benefit of trading (identified by $E_{t}\left[\boldsymbol{r}_{t+1}\right]$ ) against the predicting risk (identified by $E_{t}\left[\boldsymbol{\Sigma}_{t+1}\right]$ ) and also transaction costs. From the expressions in equation (3-4), we can see that the time-varying part of predicting risk, is indeed the CIV factor, $f_{\sigma, t}^{2}$, which characterizes the time-variation of aggregate undiversifiable risk of the whole market. The time-varying structure in volatility itself is important because it determines how the risk evolves and therefore affects the trade-off between return and risk.

\section{Optimal Mean-Variance Strategy}

The investor wants to invest in the securities for $T$ periods. The periods are numbered by $0,1, \ldots, T-1$. At time $t$, which is the beginning of period $t$, the investor knows the trading strategy in previous period, $\boldsymbol{x}_{t-1}$, the return factors at time $t, \boldsymbol{f}_{r, t}$, the CIV factor at time $t, f_{\sigma, t}$, and chooses a trading strategy $\boldsymbol{x}_{t}$ for period $t$. Meanwhile, trading is costly and the 
transaction cost (TC) associate with trading $\Delta \boldsymbol{x}_{t}=\boldsymbol{x}_{t}-\boldsymbol{x}_{t-1}$ shares takes a quadratic form,

$$
\mathrm{TC}\left(\Delta \boldsymbol{x}_{t}\right)=\frac{1}{2} \Delta \boldsymbol{x}_{t}^{T} \boldsymbol{\Lambda}_{t} \Delta \boldsymbol{x}_{t}
$$

where $\boldsymbol{\Lambda}_{t}$ is a symmetric positive-definite matrix measuring level of trading costs at time $t$, the superscript ${ }^{T}$ denotes the transpose of a matrix or a vector. ${ }^{4}$ Following GP, we further assume that transaction cost coefficient is proportional to the amount of risk,

$$
\Lambda_{t}=\lambda E_{t}\left[\Sigma_{t+1}\right]
$$

Why this special form of trading cost? The rationale lies in the dealer's compensation for risk. Consider a dealer who takes the other side of the trade $\Delta \boldsymbol{x}_{t}$, the dealer's risk is $\Delta \boldsymbol{x}_{t}^{T} \boldsymbol{\Sigma}_{t+1} \Delta \boldsymbol{x}_{t}$. Thus, the dealer's compensation for risk depends on his risk aversion level $\lambda$ and also the risk level of the market. Note that, the trading cost coefficient $\Lambda_{t}$ in our model is time-varying, and also related to the time-varying level of CIV. ${ }^{5}$ Generally speaking, the higher the CIV, the larger the transaction cost. This type of transaction cost is consistent with long standing evidence that transaction cost covary with level of volatility (see, e.g., Stoll, 1978) and also consistent with views that idiosyncratic volatility represents arbitrage cost (see, e.g. Stambaugh et al., 2015).

The investor's objective is to choose the dynamic trading strategy $\left(\boldsymbol{x}_{0}, \boldsymbol{x}_{1}, \ldots, \boldsymbol{x}_{T-1}\right)$ to maximize the present value of $T$-period future expected excess returns, penalized for risk and trading costs,

$$
\max _{\boldsymbol{x}_{0}, \boldsymbol{x}_{1}, \ldots, \boldsymbol{x}_{T-1}} E_{0}\left[\sum_{t=0}^{T-1}\left[(1-\rho)^{t+1}\left(\boldsymbol{x}_{t}^{T} \boldsymbol{r}_{t+1}-\frac{\gamma}{2} \boldsymbol{x}_{t}^{T} \boldsymbol{\Sigma}_{t+1} \boldsymbol{x}_{t}\right)-\frac{(1-\rho)^{t}}{2} \Delta \boldsymbol{x}_{t}^{T} \boldsymbol{\Lambda}_{t} \Delta \boldsymbol{x}_{t}\right]\right],
$$

\footnotetext{
${ }^{4}$ Note that quadratic form of transaction cost corresponds to a linear price impact.

${ }^{5}$ In contrast, the trading cost coefficient $\boldsymbol{\Lambda}$ in Gârleanu and Pedersen (2013) is fixed.
} 
which is equivalent to

$$
\max _{\boldsymbol{x}_{0}, \boldsymbol{x}_{1}, \ldots, \boldsymbol{x}_{T-1}} E_{0}\left[\sum_{t=0}^{T-1}\left[(1-\rho)^{t+1}\left(\boldsymbol{x}_{t}^{T} E_{t}\left[\boldsymbol{r}_{t+1}\right]-\frac{\gamma}{2} \boldsymbol{x}_{t}^{T} E_{t}\left[\boldsymbol{\Sigma}_{t+1}\right] \boldsymbol{x}_{t}\right)-\frac{(1-\rho)^{t}}{2} \Delta \boldsymbol{x}_{t}^{T} \boldsymbol{\Lambda}_{t} \Delta \boldsymbol{x}_{t}\right]\right]
$$

where $(1-\rho) \in(0,1)$ is a discount rate, $\gamma>0$ is the risk-aversion coefficient, $\boldsymbol{x}_{-1}=\mathbf{0}$. This objective function means that the investor decides his optimal trading each period by constantly balancing the expected return against the expected risk, and also transaction costs. This objective function can also be viewed as the objective of a fund manager who seeks high Sharpe ratios.

We solve the problem by dynamic programming. Define the cost-to-go function at time $t$ as follows,

$$
\begin{aligned}
& J\left(t, \boldsymbol{x}_{t-1}, \boldsymbol{f}_{r, t}, f_{\sigma, t}\right) \\
:= & \max _{\boldsymbol{x}_{t}, \ldots, \boldsymbol{x}_{T-1}} E_{t}\left[\sum_{s=t}^{T-1}\left[(1-\rho)^{s-t+1}\left(\boldsymbol{x}_{s}^{T} \boldsymbol{r}_{s+1}-\frac{\gamma}{2} \boldsymbol{x}_{s}^{T} \boldsymbol{\Sigma}_{s+1} \boldsymbol{x}_{s}\right)-\frac{(1-\rho)^{s-t}}{2} \Delta \boldsymbol{x}_{s}^{T} \boldsymbol{\Lambda}_{s} \Delta \boldsymbol{x}_{s}\right]\right] .
\end{aligned}
$$

Then, we have the following Bellman's equation,

$$
\begin{aligned}
J\left(t, x_{t-1}, \boldsymbol{f}_{r, t}, f_{\sigma, t}\right)=\max _{\boldsymbol{x}_{t}}\left\{(1-\rho) E_{t}\left[J\left(t+1, \boldsymbol{x}_{t}, \boldsymbol{f}_{r, t+1}, f_{\sigma, t+1}\right)\right]\right. \\
\left.+(1-\rho) E_{t}\left(\boldsymbol{x}_{t}^{T} \boldsymbol{r}_{t+1}-\frac{\gamma}{2} \boldsymbol{x}_{t}^{T} \boldsymbol{\Sigma}_{t+1} \boldsymbol{x}_{t}\right)-\frac{\lambda}{2} \Delta \boldsymbol{x}_{t}^{T} E_{t}\left[\boldsymbol{\Sigma}_{t+1}\right] \Delta \boldsymbol{x}_{t}\right\} .
\end{aligned}
$$

Theorem 3.1. The optimal portfolio strategy for period $t$ is given as

$$
\boldsymbol{x}_{t}^{r \sigma}=\frac{1-\rho}{a(t) \psi f_{\sigma, t}^{2}} \boldsymbol{\Omega}^{-1} \boldsymbol{C}(t) \boldsymbol{f}_{r, t}+\frac{\lambda}{a(t)} \boldsymbol{x}_{t-1}^{r \sigma},
$$

where the superscript ${ }^{r \sigma}$ represents the strategy is combing information from expected return 
and idiosyncratic volatility, and the parameters are given as follows

$$
\begin{aligned}
a(t) & =\gamma(1-\rho)+\lambda+(1-\rho) b(t), \quad b(t)=\left(\lambda-\frac{\lambda^{2}}{a(t+1)}\right) \psi, \\
\boldsymbol{C}(t) & =\boldsymbol{B}+\frac{\lambda(1-\rho)}{a(t+1)} \boldsymbol{C}(t+1)(\boldsymbol{I}-\boldsymbol{\Phi}),
\end{aligned}
$$

with $a(T-1)=\gamma(1-\rho)+\lambda, b(T-1)=0$ and $\boldsymbol{C}(T-1)=\boldsymbol{B}$.

Based on the result in Theorem 3.1, we can see that the optimal strategy trade partially from current position toward a dynamic aim portfolio, which is influenced by time-varying structure of expected return, volatility and transaction cost. ${ }^{6}$

Till now, we can clarify the differences between our formulation and that of Gârleanu and Pedersen (2013). First, in addition to return predictability, we also consider risk predictability by introducing a factor structure in volatility as shown in equations (3-4), which is absent in GP model. Second, the trading cost coefficient $\Lambda_{t}$ in our model is time-varying and also related to the time-varying risk structure through $E_{t}\left[\Sigma_{t+1}\right]$, whereas the trading cost coefficient is constant in GP model. Actually, since our model captures the time variation in volatility and the corresponding changes in transaction costs more accurately, it is able to manage the risk-exposure and the incurred transaction costs more reliably, which directly contributes to better net performance of our optimal strategy than that of GP's strategy. Thirdly, the optimal trading rate towards the aim portfolio in our model is timevarying and related to the time variation of risk and transaction costs, while, it is fixed in

\footnotetext{
${ }^{6}$ We need to emphasize that in deriving the optimal strategy, we do not make any assumption on the correlation between the return factors and the risk factor. Because in the derivation, the influences of two types of factors are perfectly separated. However, if we want to compute the cost-to-go functions explicitly, we need to specify the correlation structure between the return factors and risk factor.
} 
GP model. Finally, our dynamic portfolio selection problem is a finite horizon investment problem, whereas GP model solved the corresponding problem for an infinitely-lived agent.

\section{Understanding the Optimal Trading Rules}

We next explore the properties of the derived optimal trading strategy step by step. We first investigate how the optimal trading strategy depends solely on the return and risk predictability in the absence of transaction costs in Subsection 4.1. After that, we further investigate the impact of transaction costs in Subsection 4.2. Subsection 4.3 emphasizes the role of CIV as risk alerting index.

\subsection{Properties from return and risk predictability}

In this subsection, we investigate the properties of the optimal portfolio strategy in the absence of transaction costs, which turn out to be closely related to the classical findings of Markowitz (1952). Without transaction costs, the objective in (9) reduces to ${ }^{7}$

$$
\max _{\boldsymbol{x}_{0}, \boldsymbol{x}_{1}, \ldots, \boldsymbol{x}_{T-1}} E_{0}\left[\sum_{t=0}^{T-1}(1-\rho)^{t+1}\left(\boldsymbol{x}_{t}^{T} \boldsymbol{r}_{t+1}-\frac{\gamma}{2} \boldsymbol{x}_{t}^{T} \boldsymbol{\Sigma}_{t+1} \boldsymbol{x}_{t}\right)\right],
$$

and the optimal trading strategy takes a simple form

$$
\widetilde{\boldsymbol{x}_{t}^{*}}=\frac{1}{\gamma \psi f_{\sigma, t}^{2}} \boldsymbol{\Omega}^{-1} \boldsymbol{B} \boldsymbol{f}_{r, t} .
$$

The optimal strategy without transaction cost is intuitive and simple. The investor invests heavy into the risky assets when he expected the returns to be high (identify by $\boldsymbol{f}_{r, t}$ )

\footnotetext{
${ }^{7}$ Note that, the objective function (without transaction costs) is designed to be separable, meaning that the investor only balance the return and risk in current period to get the Sharpe ratio as high as possible. Therefore, the resulting optimal trading strategy in (13) is myopic in nature, i.e., independent of future's investment opportunity.
} 
and invests less when he expected the risk to be high (identify by $f_{\sigma, t}^{2}$ ). The investor uses his information on return, $\boldsymbol{f}_{r, t}$, to form his risky allocations in stocks, while using the risk predictor, $f_{\sigma, t}^{2}$, as a signal for the risk level of the market. Whenever there is a high level of risk signal, the investor will scale down all his positions in the risky assets simultaneously. The time-varying structure in CIV factor itself is important as it determines how the risk evolves and therefore helps timing the market. We will discuss the market timing issue at a deeper level in Section 5.1.

Recall that $E_{t}\left[\boldsymbol{r}_{t+1}\right]=\boldsymbol{B} \boldsymbol{f}_{r, t}, E_{t}\left[\boldsymbol{\Sigma}_{t+1}\right]=\psi f_{\sigma, t}^{2} \boldsymbol{\Omega}$. The optimal strategy in the absence of transaction cost can be rewritten as,

$$
\widetilde{\boldsymbol{x}_{t}^{*}}=\frac{1}{\gamma} E_{t}\left[\boldsymbol{\Sigma}_{t+1}\right]^{-1} E_{t}\left[\boldsymbol{r}_{t+1}\right]=\text { Markowitz } t
$$

which is actually the (conditional) Markowitz's tangency portfolio (leveraged by the risk aversion $\gamma$ ). It is well known that the tangency portfolio earns the highest risk adjusted return. In the absence of transaction cost, the investor would like to aim at the optimal tangency portfolio to enjoy the great Sharpe ratio. Meanwhile, the Markowitz tangency portfolio is a time-varying target in our model, since the return and risk are changing over time. With transaction cost, the investor can not re-optimize at no cost, thus needs to trade partially toward the aim. Moreover, he also needs to adjust his aim in front of current target to account for the investment opportunity in the future. This is actually the core idea behind the properties of the optimal trading strategy arising from transaction cost.

\subsection{Properties from transaction cost}

Proposition 4.1. (Trade Partially Toward the Aim) 
1) The optimal portfolio in Theorem 3.1 can be rewritten as

$$
\boldsymbol{x}_{t}^{r \sigma}=\boldsymbol{x}_{t-1}^{r \sigma}+\left(1-\frac{\lambda}{a(t)}\right)\left[a i m_{t}-\boldsymbol{x}_{t-1}^{r \sigma}\right]
$$

which implies trading at a proportional rate toward the aim portfolio,

$$
\operatorname{aim}_{t}=\frac{1-\rho}{(a(t)-\lambda) \psi f_{\sigma, t}^{2}} \boldsymbol{\Omega}^{-1} \boldsymbol{C}(t) \boldsymbol{f}_{r, t}
$$

2) The proportional rate, called "trading rate",

$$
\left(1-\frac{\lambda}{a(t)}\right)
$$

is increasing in risk aversion $\gamma$, decreasing in transaction costs parameter $\lambda$, and increasing in CIV signal persistence parameter $\psi$.

3) The optimal portfolio can also be viewed as an exponentially weighted average of current and past aim portfolios,

$$
\boldsymbol{x}_{t}^{r \sigma}=\sum_{s=0}^{t} \prod_{j=s+1}^{t}\left(\frac{\lambda}{a(j)}\right)\left(1-\frac{\lambda}{a(s)}\right) \text { aim }_{s} .
$$

From the proposition, we can rewrite the optimal portfolio as a weighted average of the current portfolio $\boldsymbol{x}_{t-1}^{r \sigma}$ and the aim portfolio:

$$
\boldsymbol{x}_{t}^{r \sigma}=\frac{\lambda}{a(t)} \boldsymbol{x}_{t-1}^{r \sigma}+\left(1-\frac{\lambda}{a(t)}\right) \operatorname{aim}_{t} .
$$

In the absence of transaction cost $(\lambda=0)$, the optimal portfolio would be the aim portfolio. With transaction cost, the investor needs to economize on trading and thus trade at a proportional rate toward the aim. The proportional rate, which is called trading rate, naturally higher if the transaction cost $(\lambda)$ is smaller. Also, the trading rate is greater if risk aversion $(\gamma)$ is larger, since a larger risk aversion makes the risk of deviating from the aim more painful. 
It is worth to mention that the trading rate also depends on the persistence of risk signal $(\psi)$, but independent on the return signal. Put differently, the return signal, $\boldsymbol{f}_{r, t}$, affects the vector components of the aim portfolio, while the persistence of risk signal affects how far the investor deviate away from the aim portfolio. The larger $\psi$, the more stable the risk signal, therefore, the higher trading rate. In other words, a more stable risk signal makes the investor trade more confidently toward the aim portfolio.

Next, we want to understand the aim portfolio. The aim portfolio turns out to be closely related to the optimal Markowitz tangency portfolio in the absence of transaction costs, which is given by equation (13) in previous subsection.

Proposition 4.2. (Aim in Front of the Target)

1) The aim portfolio is the weighted average of the current optimal tangency portfolio and a term related to future aim portfolio, i.e.,

$$
\operatorname{aim}_{t}=z(t) \widetilde{\boldsymbol{x}_{t}^{*}}+(1-z(t)) \mathcal{E}_{t}\left[\operatorname{aim}_{t+1}\right]
$$

with $\mathcal{E}_{t}\left[\operatorname{aim}_{t+1}\right]=\frac{1-\rho}{(a(t+1)-\lambda) \psi^{2} f_{\sigma, t}^{2}} \boldsymbol{\Omega}^{-1} \boldsymbol{C}(t+1)(\boldsymbol{I}-\boldsymbol{\Phi}) \boldsymbol{f}_{r, t}{ }^{8}$

2) The weighting coefficient, $z(t)=\frac{\gamma}{\gamma+b(t)}$, is decreasing in transaction cost parameter $\lambda$ (for $t \leq T-2)$ and increasing in time $t$.

3) The aim portfolio can also be rewritten as a weighted sum of the terms related to all the optimal tangency portfolios in the future,

$$
\operatorname{aim}_{t}=\sum_{s=t}^{T-1} \prod_{j=t}^{s-1}(1-z(j)) z(s) \mathcal{E}_{t}\left[\widetilde{\boldsymbol{x}_{s}^{*}}\right]
$$

\footnotetext{
${ }^{8} \mathcal{E}_{t}[\cdot]$ can be considered as an approximate expectation operator, under which we have $\mathcal{E}_{t}\left[f_{\sigma, s}^{-2} \boldsymbol{f}_{r, s}\right]=$ $\psi^{s-t} f_{\sigma, t}^{-2}(\boldsymbol{I}-\mathbf{\Phi})^{s-t} \boldsymbol{f}_{r, t}$ for $s>t$. The operator reduces to the exact expectation operator $E_{t}[\cdot]$ when $\boldsymbol{f}_{r, t}$ and $f_{\sigma, t}$ are independent and $E_{t}\left[f_{\sigma, t+1}^{-2}\right]=\psi f_{\sigma, t}^{-2}$.
} 
with $\mathcal{E}_{t}\left[\widetilde{\boldsymbol{x}_{s}^{*}}\right]=\frac{1}{\gamma \psi^{(s-t+1)} f_{\sigma, t}^{2}} \boldsymbol{\Omega}^{-1} \boldsymbol{B}(\boldsymbol{I}-\boldsymbol{\Phi})^{s-t} \boldsymbol{f}_{r, t}$.

We need to point out that although the expressions in Proposition 4.2 are quite similar to the ones in Gârleanu and Pedersen (2013), the terms related to the future are no longer simple conditional expectations.

In the absence of transaction cost, $z(t)=1$, $\operatorname{aim}_{t}=\widetilde{\boldsymbol{x}_{t}^{*}}$, the aim portfolio is indeed the optimal tangency portfolio, which earns the highest risk adjusted return. Transaction cost implies that the investor must consider the portfolio's optimality both currently and in the future. In other words, he needs to adjust his aim in front of the target to account for the investment opportunity in the future. When transaction cost is high, it is naturally to adjust the aim much further in front of the target to account for future investment opportunity (identified by a small $z(t)$ ). Meanwhile, as time approaches to the end of investment period, the investor put more and more weights on the current optimal tangency portfolio.

We conclude this subsection by emphasizing that the nature of the optimal trading strategies are fundamentally different in the cases with and without transaction costs. The optimal strategy in the absence of transaction cost, indeed, is a myopic strategy, which is independent of future evolution of risk and return. However, adding transaction cost makes the optimal strategy is forward looking. Transaction cost implies that an investor must consider the portfolio's optimality both currently and in the future.

\subsection{CIV as risk alerting signal}

This paper aims to answer how the optimal trading strategy influenced by assets' return predictability, risk predictability, and transaction costs. The general trading rules arising from transaction costs, such as trading partially toward an aim portfolio, still preserve in 
our optimal trading strategy. Next, we will focus on the new implications in our optimal trading strategy driven by risk predictability.

In order to clarify the extra role played by risk predictor, CIV, we need to understand the real, macroeconomic risk that is proxied by CIV. Existing studies in the literature have already linked CIV with many variables, ranging from growth opportunity (Bekaert et al., 2012), to household income risk (Herskovic et al., 2016), to average firm-level leverage (Van Der Heijden et al., 2017). Taken together, we believe that CIV may reflect the market wide uncertainty about the economy that affects all the risky assets. ${ }^{9}$ We further investigate such view by testing the ability of CIV to explain/predict the U.S. market portfolio's return and variance. In order to do the test, we need to compute the CIV index of U.S. market first.

Following Ang et al. (2006), we perform time series regressions at the daily frequency $(t)$ separately for every month $(m)$ of daily excess returns onto the daily returns of the Fama-French three factors,

$$
r_{m, t}^{i}=a_{m}^{i}+\beta_{i, m}^{M K T} M K T_{m, t}+\beta_{i, m}^{S M B} S M B_{m, t}+\beta_{i, m}^{H M L} H M L_{m, t}+u_{m, t}^{i} .
$$

We measure stock $i$ 's idiosyncratic volatility in month $m, \mathrm{IVOL}_{i, m}$, as the standard deviation of residual return in month $m$. We then obtain the CIV index in month $m$ by taking the (value-weighted) average of idiosyncratic volatilities of all stocks traded in U.S. market. ${ }^{10}$

Table 1 shows the contemporaneous or predictive time-series regressions for market return

\footnotetext{
${ }^{9}$ Although the two words of risk and uncertainty contain different meanings in Knight (2012)'s definitions, we use them indiscriminately in this article.

${ }^{10}$ There are usually two classical ways to do the average, equal-weighted or value-weighted. We also constructed the equal-weighted version of CIV, and found that our main results still hold. Due to space limit, we only report the value-weighted version results here.
} 
and variance with CIV as independent variable. Column (1) in Panel A reports a contemporaneous OLS regression for the market standard deviation respect to CIV. The coefficient of CIV is strong and significant, and the $R^{2}$ is $35 \%$, which indicates that CIV accounts for $35 \%$ of the variation in market variance, while Column (2-3) show that the lagged std (control variables) only accounts for $19 \%$.

Next, we analyze the ability of CIV to predict future market variance and future market return in Panel B and Panel C as shown in Table 1. Column (4) of Panel B reports a predictive OLS regression for market variance on CIV. Positive and significant coefficient indicates that CIV predicts higher market variance in the next month. The $R^{2}$ of the regression is up to $23 \%$. In Column (5-6), we control for the lagged market variance and its previous one-year average. CIV remains significant predictors of future market variance. Column (7) of Panel $\mathrm{C}$ reports a predictive OLS regression for market return on CIV. Negative (and slightly significant) coefficient means that CIV predicts lower market return in the next month. Taken evidence together, we find that CIV predicts lower future market return and higher future market variance. In other words, when CIV goes up, investment opportunities deteriorate, therefore, the investor should reduce their investment in risky assets, which is exactly our optimal strategy suggested. CIV index turn out to be a reliable risk predictor for future investment opportunity.

[Insert Table 1 here]

Now, we can summarize the new implications of our model from the perspective of CIV as risk alerting signal. First, whenever there is a high level of CIV, which signals a bad investment opportunity, the investor will scale down all his optimal positions in the risky 
assets simultaneously (identify by the fact that $\boldsymbol{x}_{t}^{r \sigma}$ is a decreasing function of $f_{\sigma, t}^{2}$ in Theorem 3.1 and also the following empirical findings). Meanwhile, a high level of CIV also represents a high level of transaction cost, resulting in a more stable trading strategy (identify by the lower trading volume during high level of CIV period as shown in Figure 5(b)). Thirdly, the investor would trade more confidently to profit from their return information with a more stable risk signal (identify by the fact that trading rate toward aim portfolio is an increasing function of $\psi$ in Proposition 4.1 and also the following empirical findings).

\section{Empirical Analysis}

In this section, we bring model to data to explore its real performance. We consider two applications: i) investing in the U.S. value-weighted market portfolio $(N=1)$; ii) investing in U.S. five industry portfolios ( $N=5$ ), including Cnsmr (C), Manuf (Ma), HiTec (Hi), Hlth $(\mathrm{Hl})$, Other $(\mathrm{O})$. The daily return of the U.S. value-weighted market portfolio is obtained from the Centre for Research in Security Prices (CRSP), which ranges from Jan. 1, 1980 to

Dec. 31, 2019 (40 years). The daily returns of the U.S. five industry portfolios are obtained from Kenneth R. French's data library website, which also range from Jan. 1, 1980 to Dec. 31, 2019.

\subsection{Market timing without transaction cost}

In order to isolate the impact of CIV from that of transaction cost, we consider a simple market timing problem without transaction cost in this subsection. Transaction cost would be added back in subsection 5.2 . 


\subsubsection{Model calibration}

We choose the Fama-French market factor, MKT, as the return predictable factor, i.e., $\boldsymbol{f}_{r, t}=\left(1, M K T_{t}\right)$, and choose CIV as the risk predictable factor, i.e., $f_{\sigma, t}=C I V_{t} \cdot{ }^{11}$ Data on prices, daily returns, and shares outstanding of all stocks in U.S. market are obtained from CRSP, which range from Jan. 1, 1980 to Dec. 31, 2019. We further assume the risk aversion coefficient $\gamma$ is $1 \times 10^{-9}$ and the discount factor is $1-\rho=0.999 .{ }^{12}$ We further assume rebalancing position monthly.

We split the 40 years data into estimation sample and testing sample by using the first 6 years data to estimate the return-risk factor parameters, and using the remaining 34 years data to test the out-of-sample performance. More specifically, we use the monthly data from Jan. 1980 to Dec. 1985 to estimate the parameters in return-risk factor model (1-4), including the factor loadings on return predictability factors, $\boldsymbol{B}_{1 \times 2}$, the parameters in the factor structure for idiosyncratic volatilities, $k_{M}$ and $\ell_{M}$, the mean-reversion coefficients of factors, $\boldsymbol{\Phi}_{2 \times 2}$ and $\psi$.

Some reader may notice that, this preliminary analysis is a little bit unrealistic by fixing the parameter estimates throughout the sample. The investor can always re-estimate the parameters after observing new data during the 34 years testing periods. Therefore, we conduct the empirical analysis in subsection 5.2 and 5.3 by a more natural rolling window

\footnotetext{
${ }^{11}$ Compared with the volatility forecasting, we recognize that it is a hard task to forecast the return. There may be other more appropriate predictors besides FF3 factors, but as we mentioned earlier, the main task of this paper is to focus on the impact of idiosyncratic volatility prediction on dynamic investment.

${ }^{12}$ Following Gârleanu and Pedersen (2013), the value of the risk aversion coefficient is corresponding to a relative risk aversion of an agent with $\$ 1$ billion under management. The value of the discount factor is quite close to the average monthly discount factor from Jan. 1980 to Dec. 2019.
} 
scheme to update the estimations.

\subsubsection{Optimal and benchmark strategies}

After estimating these parameters, the investor plug those estimations into portfolio selection model to get the optimal strategy, $x_{t}^{r \sigma}$, which combing conditional information both on expected return and on idiosyncratic volatility,

$$
x_{t}^{r \sigma}=\frac{1}{\gamma \psi f_{\sigma, t}^{2}} e^{-2 k_{M}-2 \ell_{M}^{2}} \boldsymbol{B} \boldsymbol{f}_{r, t}
$$

To split the benefits to timing idiosyncratic volatility and benefits to timing expected return, we further consider two benchmark strategies. One is the timing expected return strategy, $x_{t}^{r}$, which only uses conditional information on expected returns, but not idiosyncratic volatility (indeed it is the GP strategy). The other is a baseline strategy, $x_{t}^{b}$, which is the optimal strategy that does not do any timing. The derivation of these two types of strategies under general case (that is investing in $N$ risky assets with transaction cost) are given in Appendix E and F. By letting $\lambda=0$ and $N=1$, we obtain the following specific benchmark strategies,

$$
x_{t}^{b}=\frac{1}{\gamma\left(\sigma_{0}^{M}\right)^{2}} \boldsymbol{B} \boldsymbol{f}_{r, 0}, \quad x_{t}^{r}=\frac{1}{\gamma\left(\sigma_{0}^{M}\right)^{2}} \boldsymbol{B} \boldsymbol{f}_{r, t},
$$

where $\boldsymbol{f}_{r, 0}$ is the return predictability factors observed just before the investment, $\sigma_{0}^{M}$ is the idiosyncratic volatility of the market portfolio estimated based on the daily data during the first 6 years. ${ }^{13}$

The three types of optimal strategies and their trading volumes are presented in Figure 1. Note that the baseline strategy $x_{t}^{b}$ (the green dash line) is a buy and hold type strategy.

\footnotetext{
${ }^{13}$ Note that, $\sigma_{0}^{M}$ can also be estimated by using the daily data in the month just before the investment, in which case the main results still hold.
} 
Meanwhile, the timing expected return strategy, $x_{t}^{r}$ (the blue dash-dot line), is designed to take advantage of the time-variation structure in expected return, and our optimal strategy, $x_{t}^{r \sigma}$ (the red solid line), is designed to timing idiosyncratic volatility as well as timing expected return. The large differences between our optimal strategy and the timing expected return strategy arise in large spikes of CIV. Comparing with the timing expected return strategy, our optimal strategy invests less (more) and trade less (more) frequently in the risky market during high (low) CIV periods. Our following empirical testing further confirms that the better performance of our optimal policy mainly comes from timing CIV.

\subsubsection{Comparisons between strategies}

To examine the out-of-sample performances of a given strategy, we use the accumulated wealth, cumulative risk penalty and objective as performance measures,

$$
\begin{aligned}
& \text { wealth }_{t}=\sum_{j=0}^{t-1} x_{j} P_{j} r_{j+1}^{M}\left(1+r_{f}\right)^{t-j-1}, \\
& \text { cumulative risk penalty }_{t}=\frac{\gamma}{2} \sum_{j=0}^{t-1} x_{j}^{2}\left(\sigma_{j+1}^{M}\right)^{2}, \\
& \text { objective }_{t}=\text { wealth }_{t}-\text { cumulative risk penalty }_{t},
\end{aligned}
$$

where $P_{j}$ is the price of the market portfolio at the beginning of month $j$ (we set $P_{0}=1$ ), $r_{f}$ is the constant risk-free return, $r_{j+1}^{M}$ is the excess return of the U.S. value-weighted market portfolio, $\sigma_{j+1}^{M}$ is the realized idiosyncratic volatility of the value-weighted market portfolio.

The out-of-sample performances of the three strategies are reported in Figure 2. Under the above three measures, our optimal strategy out performs the other two benchmarks. Take a close look at the holding positions and trading volumes as presented in Figure 1, we find that the benefits mainly arise from timing the time-variation in CIV. Comparing with 
the timing expected return strategy (the blue dash-dot line), our optimal strategy (the red solid line) has small investment in the market and also small trading volumes during high level of CIV ${ }^{14}$ In contrast, if the market is at low level of CIV, the investor will adopt a more aggressive strategy by investing more in risky assets and trading more frequently to seize the good investment opportunity. Moreover, our optimal strategy will continue to outperform the timing expected return strategy in the presence of transaction cost, because our strategy has low trading volume during high trading cost periods (i.e., high CIV periods).

\section{[Insert Figure 1 here ]}

[Insert Figure 2 here ]

However, our optimal strategy runs contrary to conventional wisdom: It takes relatively less risk in recessions and crises, since CIV usually takes high levels in recessions and crises. For example, in the aftermath of the sharp price declines in the fall of 2008 (zoom in Figure 3), a widely held view was that those that reduced positions in equities were missing a once-in-a-generation buying opportunity. Basically, the timing expected return strategy also suggests to invest heavy in the market in the beginning of 2009. Yet, our strategy almost cash out and return to the market only as the spike in CIV receded. The rationality behind our strategy lies in the nature of CIV. As we already demonstrated in Table 1, CIV predicts lower future market return and higher future market variance. In other words, high CIV predicts investment opportunity deteriorating, thus worsening the investor's risk-return trade-off. Such influence of CIV on trading strategy may provide a new channel to explore

\footnotetext{
${ }^{14}$ Our findings here are consistent with Moreira and Muir (2017), who show that there are gains to moving out of the market in response to an increase in market variance.
} 
the idiosyncratic volatility puzzle.

[Insert Figure 3 here ]

Till now, we have used a simple market timing analysis to show the risk altering role of CIV index in the absence of transaction cost, and we have demonstrated that the predictability from CIV index indeed guide the investor to avoid unrewarding risk and thus achieving better performance.

\subsection{Market timing with transaction cost}

In this subsection, we consider the market timing problem with transaction cost. We assume the transaction cost coefficient $\lambda$ is $2 \times 10^{-6} \cdot{ }^{15}$ Note that, assuming $\lambda$ to be a constant does not mean the transaction cost is constant. In our model, transaction cost is time changing and related to CIV through formula, $\boldsymbol{\Lambda}_{t}=\lambda E_{t}\left[\boldsymbol{\Sigma}_{t+1}\right]=\lambda \boldsymbol{\Omega} \psi C I V_{t}^{2}$. Our following empirical test demonstrates that timing CIV can increase (net cost) Sharpe ratio because CIV can guide the investor to avoid unrewarding risk and the corresponding high trading cost.

\footnotetext{
${ }^{15}$ Collin-Dufresne et al. (2019) estimated the transaction cost coefficient based on a proprietary execution data from the historical order databases of a large investment bank. They found that the transaction cost is around $2 \times 10^{-10} Q^{2}$, where $Q$ is the dollar size of the order. As the average value of the idiosyncratic volatility of the value-weighted market portfolio is around $10^{-2}$ and the initial price of the value-weighted market portfolio is assume to be 1 dollar, we choose $\lambda=2 \times 10^{-6}$ in order to generate a similar level of transaction cost to Collin-Dufresne et al. (2019).
} 


\subsubsection{Model calibration}

Different from the procedure of estimating parameters in previous subsection 5.1, we estimate these parameters in this subsection by a rolling window scheme. Specifically, we use a 72-months-window to estimate these parameters, $\boldsymbol{B}, k_{M}, \ell_{M}, \boldsymbol{\Phi}$ and $\psi$. After estimating parameters, the investor is assume to conduct dynamic investment practices with different investment horizon, ranging from 12 months, 24 months, to 36 months. Take the 12 months investment practices for example, we estimate parameters from the 72-months-window data, plug them into portfolio selection model to get the optimal strategy, and then record the realized wealth, accumulated risk penalty and transaction costs during the 12 months investment horizon. We then repeat this process by moving the window one month ahead till touching the end of data set, that is Dec. 31, 2019. The whole process gives 396 realizations in total for 12 months investments. The numbers are 384 realizations and 372 realizations, for 24 months case and 36 months case, respectively.

\subsubsection{Optimal and benchmark strategies}

Once again, we consider three different strategies, baseline strategy, $x_{t}^{b}$, timing expected return strategy, $x_{t}^{r}$, and our optimal strategy, $x_{t}^{r \sigma}$,

$$
\begin{aligned}
x_{t}^{b} & =\frac{1-\rho}{c(t)\left(\sigma_{0}^{M}\right)^{2}} \boldsymbol{G}(t) \boldsymbol{f}_{r, 0}+\frac{\lambda}{c(t)} x_{t-1}^{b}, \\
x_{t}^{r} & =\frac{1-\rho}{c(t)\left(\sigma_{0}^{M}\right)^{2}} \boldsymbol{D}(t) \boldsymbol{f}_{r, t}+\frac{\lambda}{c(t)} x_{t-1}^{r}, \\
x_{t}^{r \sigma} & =\frac{1-\rho}{a(t) \psi f_{\sigma, t}^{2}} e^{-2 k_{M}-2 \ell_{M}^{2}} \boldsymbol{C}(t) \boldsymbol{f}_{r, t}+\frac{\lambda}{a(t)} x_{t-1}^{r \sigma},
\end{aligned}
$$

where the parameters $c(t), \boldsymbol{D}(t), \boldsymbol{G}(t)$ are given in Appendix E and $\mathrm{F}, \boldsymbol{f}_{r, 0}$ is the return predictability factors observed just before the investment, $\sigma_{0}^{M}$ is the idiosyncratic volatility 
of the market portfolio estimated based on the daily data in previous 72 months before the investment. We need to point out that with transaction cost, the baseline strategy is no longer a buy and hold type strategy. Because applying the buy and hold strategy would generate a very large lump-sum price impact at time 0. Actually, in the presence of transaction cost, all three types policies as shown in Figure 5(a) and 6(a) are accumulating risky holding slowly, therefore reducing the price impact.

\subsubsection{Comparisons between strategies}

Under the rolling window scheme, we redefine the out-of-sample performance measures, terminal wealth, cumulative risk penalty, cumulative transaction cost and objective, by their average values across all investment practices with the same length of investment horizon. Take the investment practice with 12 months horizon for example, we use the average values across the 396 realizations to measure the out-of-sample performance. Besides these measures, we also consider the Sharpe ratio with (and without) transaction cost, which are computed based on the (net of cost) terminal wealth levels of all investment practices with the same length of investment periods. We also redefine the average holding position and average trading volume, which are the average values across all periods in a single dynamic investment practice and across all investment practices with the same length of investment periods.

The out-of-sample performances of the three strategies are summarized in Table 2. Comparing to the baseline strategy and to the timing expected return strategy, our optimal strategy achieves better performance in terms of a much larger terminal wealth, a much higher Sharpe ratio, and much smaller cumulative risk penalty and cumulative transaction 
cost. We also find very large gains from idiosyncratic volatility timing, which we measured by the additional benefit of switching from timing return strategy to our fully optimal strategy (which conditions on expected returns as well as idiosyncratic volatility). Figure 4 further illustrate relative contributions from timing return and from timing idiosyncratic volatility in terms of terminal wealth, cumulative risk penalty and cumulative transaction cost. Due to timing idiosyncratic volatility, our optimal strategy is able to manage the risk-exposure and the incurred transaction costs more reliably with a large scale of reduction in risk penalty and transaction cost. Taking the 24 months investment case (as shown in Figure 4(b)) for example, switching from baseline strategy (which does not do any timing) to timing expected return strategy will result in better performance in terms of higher terminal wealth, smaller risk penalty and transaction cost, as showed by the blue pillars. Moreover, switching from timing expected return strategy to our fully optimal strategy contributes additional $15 \%$ increment in terminal wealth, additional $52 \%$ reduction in risk penalty and additional $41 \%$ reduction in transaction cost. In terms of Sharpe ratio, our optimal strategy performs even better. As shown in last two columns in Table 2, timing idiosyncratic volatility contributes $95 \%, 89 \%, 81 \%$ of the total gain of switching from the baseline strategy to our fully optimal strategy. In this sense, ignoring variation in idiosyncratic volatility is very costly and the benefits of timing idiosyncratic volatility are larger than the benefits of timing expected returns. ${ }^{16}$

\footnotetext{
${ }^{16}$ Some readers may argue that the result that timing idiosyncratic volatility is more important than timing expected returns heavily depends on our set up. In other words, FF3 may do not predict returns well. Once again, we emphasize that this paper focuses on the economic value of timing volatility, in stead of that of predicting returns.
} 
[Insert Table 2 here]

[Insert Figure 4 here]

The key reason behind net performance of our optimal strategy is from timing idiosyncratic volatility. By timing CIV (the common fluctuations of idiosyncratic volatility), the investor actually manage to switch between two types of strategies: one is conservative, the other is aggressive. When CIV is high, the investor will take the conservative type strategy by scaling down his investment in the market to avoid unrewarding risk and also trading less frequently to economize on the transaction cost. In contrast, when CIV is at low level, it is optimal to take a more aggressive type strategy by investing heavy and trading more frequently to seize the opportunity. One typical conservative type strategy is illustrated in Figure 5, which represents a 24 months dynamic investment from Jan. 2000 to Dec. 2001. Since CIV is high (meanwhile the trading cost is expensive), the investor invest less and trade less frequently to decrease risk penalty, decrease transaction cost, thus, achieve a better net performance. In contrast, when CIV is at low level (one typical example is illustrated in Figure 6), the investor takes the aggressive type strategy. By investing heavily and trading frequently, our optimal strategy (the red solid line) accumulates very larger terminal wealth at the cost of high risk penalty and transaction cost, but still achieves better net performances in terms of objective.

[Insert Figure 5 here]

[Insert Figure 6 here] 


\subsubsection{Influence of $\psi$}

In Section 4.3, we have proved that when $\psi$ is larger (i.e., the CIV signal is more persistent), the trading rate toward aim portfolio becomes larger. Now, we want to empirically confirm such influence of parameter $\psi$ from real data. We first compute the average trading rate over the $12(24,36)$ months investment periods, then plot those average trading rates respect to $\psi$ (estimated in each dynamic investment practice), as shown in Figure 7. We can see that the trading rate is indeed an increase function of $\psi$, which further confirms our previous theoretical result as shown in Proposition 4.1.

\section{[Insert Figure 7 here]}

\subsection{Testing allocation performance by industry investment}

\subsubsection{Model calibration}

In this subsection, we consider to invest in the five industry portfolios $(N=5)$, including Cnsmr (C), Manuf (Ma), HiTec (Hi), Hlth (Hl), Other $(\mathrm{O})$. The holding position is also changed monthly. We choose FF3 factor $\boldsymbol{f}_{r, t}=\left(1, M K T_{t}, S M B_{t}, H M L_{t}\right)^{T}$ as the return predictable factors and choose $f_{\sigma, t}=C I V_{t}$ as the risk predictable factor. We also assume the risk aversion coefficient $\gamma$ is $1 \times 10^{-9}$, the discount factor is $1-\rho=0.999$ and the transaction cost coefficient $\lambda$ is $2 \times 10^{-6}$.

Similar to Section 5.2, we estimate the factor loadings on return predictability factors, $\boldsymbol{B}_{5 \times 4}$, the parameters in the factor structure for idiosyncratic volatilities, $\boldsymbol{k}_{5 \times 1}$ and $\boldsymbol{\ell}_{5 \times 1}$, the mean-reversion coefficients, $\boldsymbol{\Phi}_{4 \times 4}$ and $\psi$, according to models (1) to (4) with a rolling window scheme. After estimating these parameters based on previous 72 months data in the end of each month, the investor is assume to conduct dynamic investment practices for 12 months, 
24 months and 36 months.

\subsubsection{Optimal and benchmark strategies}

Once again, we consider three different strategies, baseline strategy, $\boldsymbol{x}_{t}^{b}$, timing expected return strategy, $\boldsymbol{x}_{t}^{r}$, and our timing idiosyncratic volatility strategy, $\boldsymbol{x}_{t}^{r \sigma}$,

$$
\begin{aligned}
\boldsymbol{x}_{t}^{b} & =\frac{1-\rho}{c(t)} \boldsymbol{\Sigma}_{0}^{-1} \boldsymbol{G}(t) \boldsymbol{f}_{r, 0}+\frac{\lambda}{c(t)} \boldsymbol{x}_{t-1}^{b}, \\
\boldsymbol{x}_{t}^{r} & =\frac{1-\rho}{c(t)} \boldsymbol{\Sigma}_{0}^{-1} \boldsymbol{D}(t) \boldsymbol{f}_{r, t}+\frac{\lambda}{c(t)} \boldsymbol{x}_{t-1}^{r}, \\
\boldsymbol{x}_{t}^{r \sigma} & =\frac{1-\rho}{a(t) \psi f_{\sigma, t}^{2}} \boldsymbol{\Omega}^{-1} \boldsymbol{C}(t) \boldsymbol{f}_{r, t}+\frac{\lambda}{a(t)} \boldsymbol{x}_{t-1}^{r \sigma},
\end{aligned}
$$

where the parameters $c(t), \boldsymbol{D}(t), \boldsymbol{G}(t)$ are given in Appendix E and F, $\boldsymbol{f}_{r, 0}$ is the return predictability factors observed just before the investment, $\boldsymbol{\Sigma}_{0}$ is the covariance matrix estimated based on the daily data in previous 72 months just before the dynamic investment practice.

\subsubsection{Comparisons between strategies}

The out-of-sample performances of three strategies are summarized in Table 3. The main finding is consistent with the market timing case. Once again, by timing the volatility structure and the corresponding time variation of transaction cost, our optimal strategy achieves much larger terminal wealth, much smaller cumulative risk penalty and transaction cost, which results in a better objective. Furthermore, our optimal strategy also achieves the best Sharpe ratio with (or without) transaction cost among all three strategies. Figure 8 further collects the absolute and relative Sharpe ratio improvements both in market timing case $(N=1)$ and in industry allocation case $(N=5)$ into one figure to get a full view. Once again, blue bars represents benefits from timing expected return, and red bars repre- 
sents additional benefits from timing idiosyncratic volatility. We can see that the benefits of timing idiosyncratic volatility are significantly larger than the benefits of timing expected returns. Take 12 months investment horizon case for example, switching from baseline strategy to our optimal strategy (which conditions both on expected return and idiosyncratic volatility) will gain 0.28 (0.36 for $N=5)$ absolute increment in net Sharpe ratio. Timing idiosyncratic volatility (switching from timing expected return strategy to our optimal strategy) contributes $95 \%$ ( $64 \%$ for $N=5$ ) of the total gain, while timing expected return (switching from baseline strategy to timing expected return strategy) only contributes $5 \%$ (36\% for $N=5$ ). Therefore, the benefits from timing idiosyncratic volatility is larger than benefits from timing expected return, which is robust with different investment length, and also robust in asset allocation with single asset or multiple assets.

[Insert Table 3 here]

[Insert Figure 8 here]

\section{Conclusion}

In this paper, we derive a closed-form solution to a multi-period portfolio selection problem taking into account time-varying expected returns, time-varying idiosyncratic volatility and also time-varying transaction cost. Our optimal trading strategy indicates that the investor should trade partially from current position toward a dynamic aim portfolio, which is a weighted average of the expected future Markowitz's tangency portfolios. Our analysis offers several new insights to dynamic trading literature by including CIV as a risk alerting index. First, our optimal trading strategy is highly affected by CIV level, while is new in the 
literature. In fact, CIV index turns out to be a reliable signal that alarms both bad investment opportunity and high level of transaction cost. Therefore, the investor should invest less and trade less frequently in risky assets during high level of CIV periods. Secondly, the investor trade more closely toward the aim portfolio with a more stable CIV signal.

We also bring our model to real data to test its out-of-sample performance. By comparing our optimal dynamic strategy to two alternatives: a baseline strategy which does not do any timing, and the GP strategy which considers time-varying expected returns, but ignores the dynamics of idiosyncratic volatility. We find that the net-of-cost performance of our dynamic trading strategy is higher than the other two alternatives and the benefit mainly comes from timing idiosyncratic volatility.

The risk alerting role played by CIV is an important new implication of our model. High level of CIV, indeed, predicts lower future market return and higher future market variance, thus a bad time to invest in stock market. As a result, our strategy runs contrary to the conventional wisdom as it takes relatively less risk in recessions and crises (which usually correspond high level of CIV), yet still earns better net cost of performance. Follow this line, the equilibrium pricing implication of CIV may shed new light on the idiosyncratic volatility puzzle.

\section{Acknowledgments}

This work was partially supported by National Natural Science Foundation of China under grant 71971083, 71931004. 


\section{Appendix A: Proof of Theorem 3.1}

Proof. We prove this by backward induction. First, at time $T-1$, we know $\boldsymbol{f}_{r, T-1}, f_{\sigma, T-1}$, $\boldsymbol{x}_{T-2}$. The investor faces the following problem,

$$
\max _{\boldsymbol{x}_{T-1}} E_{T-1}\left[(1-\rho)\left(\boldsymbol{x}_{T-1}^{T} \boldsymbol{r}_{T}-\frac{\gamma}{2} \boldsymbol{x}_{T-1}^{T} \boldsymbol{\Sigma}_{T} \boldsymbol{x}_{T-1}\right)-\frac{1}{2} \Delta \boldsymbol{x}_{T-1}^{T} \boldsymbol{\Lambda}_{T-1} \Delta \boldsymbol{x}_{T-1}\right]
$$

We have objective function as follows,

$$
\begin{aligned}
F\left(T-1, \boldsymbol{x}_{T-1}\right):= & -\frac{1}{2} a(T-1) \psi f_{\sigma, T-1}^{2} \boldsymbol{x}_{T-1}^{T} \boldsymbol{\Omega} \boldsymbol{x}_{T-1}+(1-\rho) \boldsymbol{x}_{T-1}^{T} \boldsymbol{C}(T-1) \boldsymbol{f}_{r, T-1} \\
& +\lambda \psi f_{\sigma, T-1}^{2} \boldsymbol{x}_{T-1}^{T} \boldsymbol{\Omega} \boldsymbol{x}_{T-2}-\frac{\lambda}{2} \psi f_{\sigma, T-1}^{2} \boldsymbol{x}_{T-2}^{T} \boldsymbol{\Omega} \boldsymbol{x}_{T-2} .
\end{aligned}
$$

where $a(T-1)=\gamma(1-\rho)+\lambda>\lambda, \boldsymbol{C}(T-1)=\boldsymbol{B}$. And the optimal strategy is

$$
\boldsymbol{x}_{T-1}^{r \sigma}=\frac{1-\rho}{a(T-1) \psi f_{\sigma, T-1}^{2}} \boldsymbol{\Omega}^{-1} \boldsymbol{C}(T-1) \boldsymbol{f}_{r, T-1}+\frac{\lambda}{a(T-1)} \boldsymbol{x}_{T-2} .
$$

Substituting the optimal strategy back, we get the cost-to-go function at time $T-1$,

$$
\begin{aligned}
& J\left(T-1, \boldsymbol{x}_{T-2}, \boldsymbol{f}_{r, T-1}, f_{\sigma, T-1}\right) \\
= & -\frac{1}{2} b(T-2) f_{\sigma, T-1}^{2} \boldsymbol{x}_{T-2}^{T} \boldsymbol{\Omega}_{T-2}+\frac{\lambda(1-\rho)}{a(T-1)} \boldsymbol{x}_{T-2}^{T} \boldsymbol{C}(T-1) \boldsymbol{f}_{r, T-1}+Q\left(T-1, \boldsymbol{f}_{r, T-1}, f_{\sigma, T-1}\right) .
\end{aligned}
$$

where $Q\left(T-1, \boldsymbol{f}_{r, T-1}, f_{\sigma, T-1}\right)$ denote the terms that do not depend on $\boldsymbol{x}_{T-2}, b(T-2)>0$.

We assume that at time $t+1$ the cost-to-go function is given by

$$
\begin{aligned}
& J\left(t+1, \boldsymbol{x}_{t}, \boldsymbol{f}_{r, t+1}, f_{\sigma, t+1}\right) \\
= & -\frac{1}{2} b(t) f_{\sigma, t+1}^{2} \boldsymbol{x}_{t}^{T} \boldsymbol{\Omega} \boldsymbol{x}_{t}+\frac{\lambda(1-\rho)}{a(t+1)} \boldsymbol{x}_{t}^{T} \boldsymbol{C}(t+1) \boldsymbol{f}_{r, t+1}+Q\left(t+1, \boldsymbol{f}_{r, t+1}, f_{\sigma, t+1}\right),
\end{aligned}
$$

where $Q\left(t+1, \boldsymbol{f}_{r, t+1}, f_{\sigma, t+1}\right)$ denote the terms that do not depend on $\boldsymbol{x}_{t}, b(t)>0$. We prove that the same structure for the optimal portfolio strategy holds for time $t$. 
At time $t$, according to Bellman's equation (11), the objective function is

$$
\begin{aligned}
F\left(t, \boldsymbol{x}_{t}\right):= & (1-\rho) E_{t}\left[J\left(t+1, \boldsymbol{x}_{t}, \boldsymbol{f}_{r, t+1}, f_{\sigma, t+1}\right)\right]+(1-\rho)\left(\boldsymbol{x}_{t}^{T} E_{t}\left[\boldsymbol{r}_{t+1}\right]-\frac{\gamma}{2} \boldsymbol{x}_{t}^{T} E_{t}\left[\boldsymbol{\Sigma}_{t+1}\right] \boldsymbol{x}_{t}\right) \\
& -\frac{\lambda}{2}\left(\boldsymbol{x}_{t}-\boldsymbol{x}_{t-1}\right)^{T} E_{t}\left[\boldsymbol{\Sigma}_{t+1}\right]\left(\boldsymbol{x}_{t}-\boldsymbol{x}_{t-1}\right) \\
= & -\frac{1}{2} a(t) \psi f_{\sigma, t}^{2} \boldsymbol{x}_{t}^{T} \boldsymbol{\Omega} \boldsymbol{x}_{t}+(1-\rho) \boldsymbol{x}_{t}^{T} \boldsymbol{C}(t) \boldsymbol{f}_{r, t}+\lambda \psi f_{\sigma, t}^{2} \boldsymbol{x}_{t}^{T} \boldsymbol{\Omega} \boldsymbol{x}_{t-1}-\frac{\lambda \psi f_{\sigma, t}^{2}}{2} \boldsymbol{x}_{t-1}^{T} \boldsymbol{\Omega} \boldsymbol{x}_{t-1} \\
& +(1-\rho) E_{t}\left[Q\left(t+1, \boldsymbol{f}_{r, t+1}, f_{\sigma, t+1}\right)\right] .
\end{aligned}
$$

It is easy to check that $a(t)>\lambda$. And the optimal strategy is

$$
\boldsymbol{x}_{t}^{r \sigma}=\frac{1-\rho}{a(t) \psi f_{\sigma, t}^{2}} \boldsymbol{\Omega}^{-1} \boldsymbol{C}(t) \boldsymbol{f}_{r, t}+\frac{\lambda}{a(t)} \boldsymbol{x}_{t-1} .
$$

Substituting the optimal strategy back, we get the cost-to-go function,

$$
J\left(t, \boldsymbol{x}_{t-1}, \boldsymbol{f}_{r, t}, f_{\sigma, t}\right)=-\frac{1}{2} b(t-1) f_{\sigma, t}^{2} \boldsymbol{x}_{t-1}^{T} \boldsymbol{\Omega} \boldsymbol{x}_{t-1}+\frac{\lambda(1-\rho)}{a(t)} \boldsymbol{x}_{t-1}^{T} \boldsymbol{C}(t) \boldsymbol{f}_{r, t}+Q\left(t, \boldsymbol{f}_{r, t}, f_{\sigma, t}\right),
$$

where $Q\left(t, \boldsymbol{f}_{r, t}, f_{\sigma, t}\right)$ also denote the terms that do not depend on $\boldsymbol{x}_{t-1}, b(t-1)>0$. Therefore, the main conclusion holds for all time $t$.

\section{Appendix B: A useful Lemma 1}

Lemma 1. The parameters have the following monotonicity properties:

1) $a(t)$ and $b(t)$ are both decreasing functions of $t$,

2) $a(t)$ is increasing in $\gamma$ (for $t \leq T-1)$,

3) a(t) is increasing in $\psi$ (for $t \leq T-2)$,

4) $b(t)$ is increasing in $\lambda$ (for $t \leq T-2)$,

5) $\left(1-\frac{\lambda}{a(t)}\right)$ is decreasing in $\lambda($ for $t \leq T-1)$. 
Proof. We first prove that $a(t)$ and $b(t)$ are both decreasing functions of $t$ by backward induction. It is easy to see that $a(T-1)=\gamma(1-\rho)+\lambda>\lambda$ and $b(T-1)=0$, which implies

$$
\begin{aligned}
& b(T-2)=\left(\lambda-\frac{\lambda^{2}}{a(T-1)}\right) \psi=\lambda \psi \frac{a(T-1)-\lambda}{a(T-1)}>0=b(T-1), \\
& a(T-2)=\gamma(1-\rho)+\lambda+(1-\rho) b(T-2)>a(T-1) .
\end{aligned}
$$

Assume that at time $t+1$, we have $a(t+1)>a(t+2)$ and $b(t+1)>b(t+2)$. Then, at time $t$, we further have

$$
a(t)-a(t+1)=(1-\rho)[b(t)-b(t+1)]=-(1-\rho) \psi \lambda^{2}\left(\frac{1}{a(t+1)}-\frac{1}{a(t+2)}\right)>0
$$

which implies $a(t)>a(t+1)$ and $b(t)>b(t+1)$. Therefore, $a(t)$ and $b(t)$ are both decreasing functions of $t$.

Next, we also prove the other four monotonicity properties by backward induction.

- $a(T-1)=\gamma(1-\rho)+\lambda$ is an increasing function of $\gamma$.

- $a(T-2)=\gamma(1-\rho)+\lambda+(1-\rho)\left(\lambda-\frac{\lambda^{2}}{a(T-1)}\right) \psi$ is an increasing function of $\psi$.

- $\frac{d b(T-2)}{d \lambda}=\frac{(a(T-1)-\lambda)^{2} \psi}{(a(T-1))^{2}}>0$ implies that $b(T-2)$ is an increasing function of $\lambda$.

- $\frac{d\left(1-\frac{\lambda}{a(T-1)}\right)}{d \lambda}=\frac{\lambda-a(T-1)}{(a(T-1))^{2}}=\frac{-\gamma(1-\rho)}{(a(T-1))^{2}}<0$ implies that $\left(1-\frac{\lambda}{a(T-1)}\right)$ is a decreasing function of $\lambda$.

Assume that these four monotonicity properties hold for time $t+1$. Then, we prove these monotonicity properties still hold for time $t$.

- As $a(t)=\gamma(1-\rho)+\lambda+(1-\rho)\left(\lambda-\frac{\lambda^{2}}{a(t+1)}\right) \psi$ and $a(t+1)$ is an increasing function of $\gamma, a(t)$ is an increasing function of $\gamma$. 
- As $a(t)=\gamma(1-\rho)+\lambda+(1-\rho)\left(\lambda-\frac{\lambda^{2}}{a(t+1)}\right) \psi, a(t+1)>\lambda$ and $a(t+1)$ is an increasing function of $\psi, a(t)$ is also an increasing function of $\psi$.

- As $\frac{d b(t)}{d \lambda}=\frac{\psi(a(t+1)-\lambda)^{2}+\psi \lambda^{2}(1-\rho) \frac{d b(t+1)}{d \lambda}}{a^{2}(t+1)}$ and $b(t+1)$ is an increasing function of $\lambda, b(t)$ is also an increasing function of $\lambda$.

- Assume that $\lambda_{2}>\lambda_{1}$. Then, we have

$$
\begin{aligned}
& \left(1-\frac{\lambda_{2}}{a\left(t ; \lambda_{2}\right)}\right)-\left(1-\frac{\lambda_{1}}{a\left(t ; \lambda_{1}\right)}\right) \\
= & \frac{\gamma(1-\rho)\left(\lambda_{1}-\lambda_{2}\right)+\lambda_{1} \lambda_{2}(1-\rho) \psi\left[\left(1-\frac{\lambda_{2}}{a\left(t+1 ; \lambda_{2}\right)}\right)-\left(1-\frac{\lambda_{1}}{a\left(t+1 ; \lambda_{1}\right)}\right)\right]}{a\left(t ; \lambda_{1}\right) a\left(t ; \lambda_{2}\right)} \\
< & 0,
\end{aligned}
$$

which implies that $\left(1-\frac{\lambda}{a(t)}\right)$ is a decreasing function of $\lambda$. The last inequality in the proof is due to the fact $\left(1-\frac{\lambda}{a(t+1)}\right)$ is a decreasing function of $\lambda$.

\section{Appendix C: Proof of Proposition 4.1}

Proof. By rewriting the optimal portfolio strategy, we can obtain the conclusion in 1).

Based on Lemma 1, we have the conclusion in 2).

Through simply mathematical derivation, we can also obtain the conclusion in 3).

\section{Appendix D: Proof of Proposition 4.2}

Proof. Let us first define an approximate expectation operator $\mathcal{E}_{t}[\cdot]$, under which we have

$$
\mathcal{E}_{t}\left[f_{\sigma, s}^{-2} \boldsymbol{f}_{r, s}\right]=\psi^{s-t} f_{\sigma, t}^{-2}(\boldsymbol{I}-\boldsymbol{\Phi})^{s-t} \boldsymbol{f}_{r, t}, \text { for } s>t
$$


Then, it is easy to check that

$$
\begin{aligned}
& \mathcal{E}_{t}\left[\operatorname{aim}_{t+1}\right]=\frac{1-\rho}{(a(t+1)-\lambda) \psi^{2} f_{\sigma, t}^{2}} \boldsymbol{\Omega}^{-1} \boldsymbol{C}(t+1)(\boldsymbol{I}-\boldsymbol{\Phi}) \boldsymbol{f}_{r, t} \\
& \mathcal{E}_{t}\left[\widetilde{\boldsymbol{x}_{s}^{*}}\right]=\frac{1}{\gamma \psi^{(s-t+1)} f_{\sigma, t}^{2}} \boldsymbol{\Omega}^{-1} \boldsymbol{B}(\boldsymbol{I}-\boldsymbol{\Phi})^{s-t} \boldsymbol{f}_{r, t}
\end{aligned}
$$

1) At time $t$, we have

$$
\begin{aligned}
& \operatorname{aim}_{t} \\
= & \frac{1-\rho}{(a(t)-\lambda) \psi f_{\sigma, t}^{2}} \boldsymbol{\Omega}^{-1} \boldsymbol{C}(t) \boldsymbol{f}_{r, t} \\
= & \frac{1-\rho}{(a(t)-\lambda) \psi f_{\sigma, t}^{2}} \boldsymbol{\Omega}^{-1} \boldsymbol{B} \boldsymbol{f}_{r, t}+\frac{1-\rho}{(a(t)-\lambda) \psi f_{\sigma, t}^{2}} \cdot \frac{\lambda(1-\rho)}{a(t+1)} \boldsymbol{\Omega}^{-1} \boldsymbol{C}(t+1)(\boldsymbol{I}-\boldsymbol{\Phi}) \boldsymbol{f}_{r, t} \\
= & \frac{\gamma(1-\rho)}{a(t)-\lambda} \text { Markowitz }_{t}+\frac{(a(t+1)-\lambda) \psi}{a(t)-\lambda} \cdot \frac{\lambda(1-\rho)}{a(t+1)} \mathcal{E}_{t}\left[\operatorname{aim}_{t+1}\right] \\
= & \frac{\gamma}{\gamma+b(t)} \text { Markowitz }_{t}+\frac{b(t)}{\gamma+b(t)} \mathcal{E}_{t}\left[\operatorname{aim}_{t+1}\right] .
\end{aligned}
$$

2) According to Lemma 1, we have the conclusion in 2).

3) The conclusion can be proved by showing that

$$
\sum_{s=t}^{T-1} \prod_{j=t}^{s-1}(1-z(j)) z(s) \gamma^{-1} \psi^{-(s-t)} \boldsymbol{B}(\boldsymbol{I}-\mathbf{\Phi})^{s-t}=\frac{1-\rho}{a(t)-\lambda} \boldsymbol{C}(t) .
$$

We prove the above expression by backward induction. Obviously, at time $T-1$, we have

$$
\sum_{s=T-1}^{T-1} \prod_{j=T-1}^{s-1}(1-z(j)) z(s) \gamma^{-1} \psi^{-(s-t)} \boldsymbol{B}(\boldsymbol{I}-\boldsymbol{\Phi})^{s-t}=\frac{1}{\gamma} \boldsymbol{B}=\frac{1-\rho}{a(T-1)-\lambda} \boldsymbol{C}(T-1) .
$$

Assume that the expression holds at time $t+1$, i.e.,

$$
\sum_{s=t+1}^{T-1} \prod_{j=t+1}^{s-1}(1-z(j)) z(s) \gamma^{-1} \psi^{-(s-t-1)} \boldsymbol{B}(\boldsymbol{I}-\boldsymbol{\Phi})^{s-t-1}=\frac{1-\rho}{a(t+1)-\lambda} \boldsymbol{C}(t+1) .
$$


Then, at time $t$, we have

$$
\begin{aligned}
& \sum_{s=t}^{T-1} \prod_{j=t}^{s-1}(1-z(j)) z(s) \gamma^{-1} \psi^{-(s-t)} \boldsymbol{B}(\boldsymbol{I}-\boldsymbol{\Phi})^{s-t} \\
= & z(t) \gamma^{-1} \boldsymbol{B}+(1-z(t)) \frac{1-\rho}{a(t+1)-\lambda} \psi^{-1} \boldsymbol{C}(t+1)(\boldsymbol{I}-\boldsymbol{\Phi}) \\
= & \frac{1}{\gamma+b(t)}\left[\boldsymbol{B}+\left(\lambda-\frac{\lambda^{2}}{a(t+1)}\right) \psi \frac{1-\rho}{a(t+1)-\lambda} \psi^{-1} \boldsymbol{C}(t+1)(\boldsymbol{I}-\boldsymbol{\Phi})\right] \\
= & \frac{1-\rho}{a(t)-\lambda}\left[\boldsymbol{B}+\frac{\lambda(1-\rho)}{a(t+1)} \boldsymbol{C}(t+1)(\boldsymbol{I}-\boldsymbol{\Phi})\right] \\
= & \frac{1-\rho}{a(t)-\lambda} \boldsymbol{C}(t) .
\end{aligned}
$$

\section{Appendix E: Derivation of baseline strategy}

When the investor does not consider the time-varying expected return and idiosyncratic volatility (i.e., does not do any timing), he just chooses,

$$
E_{s}\left[\boldsymbol{r}_{t}\right]=\boldsymbol{B} \boldsymbol{f}_{r, 0}, \quad \boldsymbol{\Sigma}_{t}=\boldsymbol{\Sigma}_{0}
$$

where $\boldsymbol{f}_{r, 0}$ is the return predictability factors observed just before the investment, $\boldsymbol{\Sigma}_{0}$ is the estimated covariance matrix just before the investment. The corresponding baseline strategy for period $t$ is given as

$$
\boldsymbol{x}_{t}^{b}=\frac{1-\rho}{c(t)} \boldsymbol{\Sigma}_{0}^{-1} \boldsymbol{G}(t) \boldsymbol{f}_{r, 0}+\frac{\lambda}{c(t)} \boldsymbol{x}_{t-1}^{b}
$$

where

$c(t)=\gamma(1-\rho)+\lambda+(1-\rho) d(t), \quad d(t)=\left(\lambda-\frac{\lambda^{2}}{c(t+1)}\right), \quad \boldsymbol{G}(t)=\boldsymbol{B}+\frac{\lambda(1-\rho)}{c(t+1)} \boldsymbol{G}(t+1)$, with $c(T-1)=\gamma(1-\rho)+\lambda, d(T-1)=0$ and $\boldsymbol{G}(T-1)=\boldsymbol{B}$. 


\section{Proof.}

Similar to the timing idiosyncratic volatility strategy, we also drive the baseline strategy by backward induction. At time $T-1$, we know $\boldsymbol{x}_{T-2}$ and have the objective function as follows,

$$
\begin{aligned}
& F\left(T-1, \boldsymbol{x}_{T-1}\right) \\
:= & -\frac{1}{2} c(T-1) \boldsymbol{x}_{T-1}^{T} \boldsymbol{\Sigma}_{0} \boldsymbol{x}_{T-1}+(1-\rho) \boldsymbol{x}_{T-1}^{T} \boldsymbol{G}(T-1) \boldsymbol{f}_{r, 0}+\lambda \boldsymbol{x}_{T-1}^{T} \boldsymbol{\Sigma}_{0} \boldsymbol{x}_{T-2}-\frac{\lambda}{2} \boldsymbol{x}_{T-2}^{T} \boldsymbol{\Sigma}_{0} \boldsymbol{x}_{T-2} .
\end{aligned}
$$

where $c(T-1)=\gamma(1-\rho)+\lambda>\lambda$ and $\boldsymbol{G}(T-1)=\boldsymbol{B}$. And the baseline strategy is

$$
\boldsymbol{x}_{T-1}^{b}=\frac{1-\rho}{c(T-1)} \boldsymbol{\Sigma}_{0}^{-1} \boldsymbol{G}(T-1) \boldsymbol{f}_{r, 0}+\frac{\lambda}{c(T-1)} \boldsymbol{x}_{T-2} .
$$

Substituting the strategy back, we get the cost-to-go function,

$$
J\left(T-1, \boldsymbol{x}_{T-2}\right)=-\frac{1}{2} d(T-2) \boldsymbol{x}_{T-2}^{T} \boldsymbol{\Sigma}_{0} \boldsymbol{x}_{T-2}+\frac{\lambda(1-\rho)}{c(T-1)} \boldsymbol{x}_{T-2}^{T} \boldsymbol{G}(T-1) \boldsymbol{f}_{r, 0}+Q(T-1) .
$$

where $Q(T-1)$ denote the terms, which do not depend on $\boldsymbol{x}_{T-2}, d(T-2)>0$.

We assume that at time $t+1$ the cost-to-go function is given by

$$
J\left(t+1, \boldsymbol{x}_{t}\right)=-\frac{1}{2} d(t) \boldsymbol{x}_{t}^{T} \boldsymbol{\Sigma}_{0} \boldsymbol{x}_{t}+\frac{\lambda(1-\rho)}{c(t+1)} \boldsymbol{x}_{t}^{T} \boldsymbol{G}(t+1) \boldsymbol{f}_{r, 0}+Q(t+1),
$$

where $Q(t+1)$ denote the terms, which do not depend on $\boldsymbol{x}_{t}$, and $d(t)>0$. We prove that the same structure for the optimal portfolio strategy holds for time $t$.

At time $t$, the objective function is

$$
\begin{aligned}
F\left(t, \boldsymbol{x}_{t}\right):= & (1-\rho) E_{t}\left[J\left(t+1, \boldsymbol{x}_{t}\right)\right]+(1-\rho)\left(\boldsymbol{x}_{t}^{T} E_{t}\left[\boldsymbol{r}_{t+1}\right]-\frac{\gamma}{2} \boldsymbol{x}_{t}^{T} E_{t}\left[\boldsymbol{\Sigma}_{t+1}\right] \boldsymbol{x}_{t}\right) \\
& -\frac{\lambda}{2}\left(\boldsymbol{x}_{t}-\boldsymbol{x}_{t-1}\right)^{T} E_{t}\left[\boldsymbol{\Sigma}_{t+1}\right]\left(\boldsymbol{x}_{t}-\boldsymbol{x}_{t-1}\right) \\
= & -\frac{1}{2} c(t) \boldsymbol{x}_{t}^{T} \boldsymbol{\Sigma}_{0} \boldsymbol{x}_{t}+(1-\rho) \boldsymbol{x}_{t}^{T} \boldsymbol{G}(t) \boldsymbol{f}_{r, 0}+\lambda \boldsymbol{x}_{t}^{T} \boldsymbol{\Sigma}_{0} \boldsymbol{x}_{t-1}-\frac{\lambda}{2} \boldsymbol{x}_{t-1}^{T} \boldsymbol{\Sigma}_{0} \boldsymbol{x}_{t-1}+(1-\rho) Q(t+1)
\end{aligned}
$$


It is easy to check that $c(t)>\lambda$. And the baseline strategy is

$$
\boldsymbol{x}_{t}^{b}=\frac{1-\rho}{c(t)} \boldsymbol{\Sigma}_{0}^{-1} \boldsymbol{G}(t) \boldsymbol{f}_{r, 0}+\frac{\lambda}{c(t)} \boldsymbol{x}_{t-1} .
$$

Substituting the strategy back, we get the cost-to-go function,

$$
J\left(t, \boldsymbol{x}_{t-1}\right)=-\frac{1}{2} d(t-1) \boldsymbol{x}_{t-1}^{T} \boldsymbol{\Sigma}_{0} \boldsymbol{x}_{t-1}+\frac{\lambda(1-\rho)}{c(t)} \boldsymbol{x}_{t-1}^{T} \boldsymbol{G}(t) \boldsymbol{f}_{r, t}+Q(t)
$$

where $Q(t)$ denote the terms, which do not depend on $\boldsymbol{x}_{t-1}, d(t-1)>0$. Thus, the main conclusion holds for all time $t$.

\section{Appendix F: Derivation of timing expected return strategy}

When the investor only considers time-varying expected return, but ignores time-varying idiosyncratic volatility, he chooses to predict the expected return but fix the variance at the initial level,

$$
E_{s}\left[\boldsymbol{r}_{t+1}\right]=\boldsymbol{B}(\boldsymbol{I}-\boldsymbol{\Phi})^{t-s} \boldsymbol{f}_{r, s}, \quad \boldsymbol{\Sigma}_{t}=\boldsymbol{\Sigma}_{0},
$$

where $\boldsymbol{\Sigma}_{0}$ is the estimated covariance matrix just before the investment. The corresponding timing expected return strategy for period $t$ is given as

$$
\boldsymbol{x}_{t}^{r}=\frac{1-\rho}{c(t)} \boldsymbol{\Sigma}_{0}^{-1} \boldsymbol{D}(t) \boldsymbol{f}_{r, t}+\frac{\lambda}{c(t)} \boldsymbol{x}_{t-1}^{r},
$$

where

$$
\begin{aligned}
& c(t)=\gamma(1-\rho)+\lambda+(1-\rho) d(t), \quad d(t)=\left(\lambda-\frac{\lambda^{2}}{c(t+1)}\right), \\
& \boldsymbol{D}(t)=\boldsymbol{B}+\frac{\lambda(1-\rho)}{c(t+1)} \boldsymbol{D}(t+1)(\boldsymbol{I}-\boldsymbol{\Phi}),
\end{aligned}
$$

with $c(T-1)=\gamma(1-\rho)+\lambda, d(T-1)=0$ and $\boldsymbol{D}(T-1)=\boldsymbol{B}$. 
Proof. Similar to the timing idiosyncratic volatility strategy, we also drive the timing expected return strategy by backward induction. At time $T-1$, we know $\boldsymbol{f}_{r, T-1}, \boldsymbol{x}_{T-2}$, and have the objective function as follows,

$$
\begin{aligned}
& F\left(T-1, \boldsymbol{x}_{T-1}\right) \\
:= & -\frac{1}{2} c(T-1) \boldsymbol{x}_{T-1}^{T} \boldsymbol{\Sigma}_{0} \boldsymbol{x}_{T-1}+(1-\rho) \boldsymbol{x}_{T-1}^{T} \boldsymbol{D}(T-1) \boldsymbol{f}_{r, T-1}+\lambda \boldsymbol{x}_{T-1}^{T} \boldsymbol{\Sigma}_{0} \boldsymbol{x}_{T-2}-\frac{\lambda}{2} \boldsymbol{x}_{T-2}^{T} \boldsymbol{\Sigma}_{0} \boldsymbol{x}_{T-2} .
\end{aligned}
$$

where $c(T-1)=\gamma(1-\rho)+\lambda>\lambda$ and $\boldsymbol{D}(T-1)=\boldsymbol{B}$. And the timing expected return strategy is

$$
\boldsymbol{x}_{T-1}^{r}=\frac{1-\rho}{c(T-1)} \boldsymbol{\Sigma}_{0}^{-1} \boldsymbol{D}(T-1) \boldsymbol{f}_{r, T-1}+\frac{\lambda}{c(T-1)} \boldsymbol{x}_{T-2} .
$$

Substituting the solution back, we get the cost-to-go function,

$$
\begin{aligned}
& J\left(T-1, \boldsymbol{x}_{T-2}, \boldsymbol{f}_{r, T-1}\right) \\
= & -\frac{1}{2} d(T-2) \boldsymbol{x}_{T-2}^{T} \boldsymbol{\Sigma}_{0} \boldsymbol{x}_{T-2}+\frac{\lambda(1-\rho)}{c(T-1)} \boldsymbol{x}_{T-2}^{T} \boldsymbol{D}(T-1) \boldsymbol{f}_{r, T-1}+Q\left(T-1, \boldsymbol{f}_{r, T-1}\right) .
\end{aligned}
$$

where $Q\left(T-1, \boldsymbol{f}_{r, T-1}\right)$ denote the terms, which do not depend on $\boldsymbol{x}_{T-2}$, and $d(T-2)>0$.

We assume that at time $t+1$ the cost-to-go function is given by

$$
J\left(t+1, \boldsymbol{x}_{t}, \boldsymbol{f}_{r, t+1}\right)=-\frac{1}{2} d(t) \boldsymbol{x}_{t}^{T} \boldsymbol{\Sigma}_{0} \boldsymbol{x}_{t}+\frac{\lambda(1-\rho)}{c(t+1)} \boldsymbol{x}_{t}^{T} \boldsymbol{D}(t+1) \boldsymbol{f}_{r, t+1}+Q\left(t+1, \boldsymbol{f}_{r, t+1}\right),
$$

where $Q\left(t+1, \boldsymbol{f}_{r, t+1}\right)$ denote the terms, which do not depend on $\boldsymbol{x}_{t}$, and $d(t)>0$. We prove that the same structure for the optimal portfolio strategy holds for time $t$. 
At time $t$, the objective function is

$$
\begin{aligned}
F\left(t, \boldsymbol{x}_{t}\right):= & (1-\rho) E_{t}\left[J\left(t+1, \boldsymbol{x}_{t}, \boldsymbol{f}_{r, t+1}\right)\right]+(1-\rho)\left(\boldsymbol{x}_{t}^{T} E_{t}\left[\boldsymbol{r}_{t+1}\right]-\frac{\gamma}{2} \boldsymbol{x}_{t}^{T} E_{t}\left[\boldsymbol{\Sigma}_{t+1}\right] \boldsymbol{x}_{t}\right) \\
& -\frac{\lambda}{2}\left(\boldsymbol{x}_{t}-\boldsymbol{x}_{t-1}\right)^{T} E_{t}\left[\boldsymbol{\Sigma}_{t+1}\right]\left(\boldsymbol{x}_{t}-\boldsymbol{x}_{t-1}\right) \\
= & -\frac{1}{2} c(t) \boldsymbol{x}_{t}^{T} \boldsymbol{\Sigma}_{0} \boldsymbol{x}_{t}+(1-\rho) \boldsymbol{x}_{t}^{T} \boldsymbol{D}(t) \boldsymbol{f}_{r, t}+\lambda \boldsymbol{x}_{t}^{T} \boldsymbol{\Sigma}_{0} \boldsymbol{x}_{t-1}-\frac{\lambda}{2} \boldsymbol{x}_{t-1}^{T} \boldsymbol{\Sigma}_{0} \boldsymbol{x}_{t-1} \\
& +(1-\rho) E_{t}\left[Q\left(t+1, \boldsymbol{f}_{r, t+1}\right)\right] .
\end{aligned}
$$

It is easy to check that $c(t)>\lambda$. And the timing expected return strategy is

$$
\boldsymbol{x}_{t}^{r}=\frac{1-\rho}{c(t)} \boldsymbol{\Sigma}_{0}^{-1} \boldsymbol{D}(t) \boldsymbol{f}_{r, t}+\frac{\lambda}{c(t)} \boldsymbol{x}_{t-1} .
$$

Substituting the strategy back, we get the cost-to-go function,

$$
J\left(t, \boldsymbol{x}_{t-1}, \boldsymbol{f}_{r, t}\right)=-\frac{1}{2} d(t-1) \boldsymbol{x}_{t-1}^{T} \boldsymbol{\Sigma}_{0} \boldsymbol{x}_{t-1}+\frac{\lambda(1-\rho)}{c(t)} \boldsymbol{x}_{t-1}^{T} \boldsymbol{D}(t) \boldsymbol{f}_{r, t}+Q\left(t, \boldsymbol{f}_{r, t}\right),
$$

where $Q\left(t, \boldsymbol{f}_{r, t}\right)$ denote the terms, which do not depend on $\boldsymbol{x}_{t-1}$, and $d(t-1)>0$. Thus, the main conclusion holds for all time $t$.

\section{References}

Ang, A., Hodrick, R. J., Xing, Y., and Zhang, X. (2006). The cross-section of volatility and expected returns. The Journal of Finance, 61(1):259-299.

Balduzzi, P. and Lynch, A. W. (1999). Transaction costs and predictability: Some utility cost calculations. Journal of Financial Economics, 52(1):47-78.

Bekaert, G., Hodrick, R. J., and Zhang, X. (2012). Aggregate idiosyncratic volatility. Journal of Financial and Quantitative Analysis, 47(6):1155-1185.

Busse, J. A. (1999). Volatility timing in mutual funds: Evidence from daily returns. Review of Financial Studies, 12(5):1009-1041.

Collin-Dufresne, P., Daniel, K., and Sağlam, M. (2019). Liquidity regimes and optimal dynamic asset allocation. Journal of Financial Economics. 
Constantinides, G. M. (1986). Capital market equilibrium with transaction costs. Journal of political Economy, 94(4):842-862.

Cui, X., Gao, J., Li, X., and Li, D. (2014). Optimal multi-period mean-variance policy under no-shorting constraint. European Journal of Operational Research, 234(2):459-468.

Ding, Y., Engle, R., Li, Y., and Zheng, X. (2020). Factor modeling for volatility. Working paper.

Duarte, J., Kamara, A., Siegel, S., and Sun, C. (2014). The systematic risk of idiosyncratic volatility. Working paper.

Fleming, J., Kirby, C., and Ostdiek, B. (2001). The economic value of volatility timing. The Journal of Finance, 56(1):329-352.

Fleming, J., Kirby, C., and Ostdiek, B. (2003). The economic value of volatility timing using "realized" volatility. Journal of Financial Economics, 67:473-509.

Gao, J. and Li, D. (2013). Optimal cardinality constrained portfolio selection. Operations research, 61(3):745761.

Gârleanu, N. and Pedersen, L. H. (2013). Dynamic trading with predictable returns and transaction costs. The Journal of Finance, 68(6):2309-2340.

Gârleanu, N. and Pedersen, L. H. (2016). Dynamic portfolio choice with frictions. Journal of Economic Theory, 165:487-516.

Glasserman, P. and Xu, X. (2013). Robust portfolio control with stochastic factor dynamics. Operations Research, 61(4):874-893.

Herskovic, B., Kelly, B., Lustig, H., and Van Nieuwerburgh, S. (2016). The common factor in idiosyncratic volatility: Quantitative asset pricing implications. Journal of Financial Economics, 119(2):249-283.

Knight, F. H. (2012). Risk, uncertainty and profit. Courier Corporation.

Li, D. and Ng, W.-L. (2000). Optimal dynamic portfolio selection: Multiperiod mean?variance formulation. Mathematical Finance, 10(3):387-406.

Li, X., Zhou, X. Y., and Lim, A. E. (2002). Dynamic mean-variance portfolio selection with no-shorting constraints. SIAM Journal on Control and Optimization, 40(5):1540-1555.

Liu, H. (2004). Optimal consumption and investment with transaction costs and multiple risky assets. The 
Journal of Finance, 59(1):289-338.

Lynch, A. W. and Balduzzi, P. (2000). Predictability and transaction costs: The impact on rebalancing rules and behavior. The Journal of Finance, 55(5):2285-2309.

Merton, R. C. (1969). Lifetime portfolio selection under uncertainty: The continuous-time case. The review of Economics and Statistics, pages 247-257.

Moreira, A. and Muir, T. (2017). Volatility-managed portfolios. The Journal of Finance, 72(4):1611-1644.

Moreira, A. and Muir, T. (2018). Should long-term investors time volatility? Working paper.

Mossin, J. (1968). Optimal multiperiod portfolio policies. The Journal of Business, 41(2):215-229.

Samuelson, P. (1969). A lifetime portfolio selection by dynamic stochastic programming. Review of Economics and Statistics, 5(3):239-46.

Stambaugh, R. F., Yu, J., and Yuan, Y. (2015). Arbitrage asymmetry and the idiosyncratic volatility puzzle. The Journal of Finance, 70(5):1903-1948.

Stoll, H. R. (1978). The supply of dealer services in securities markets. The Journal of Finance, 33(4):11331151.

Van Der Heijden, T., Zeng, Q., and Zhu, Y. (2017). A three-factor model of idiosyncratic volatility. Working paper.

Zhang, J., Jin, Z., and An, Y. (2017). Dynamic portfolio optimization with ambiguity aversion. Journal of Banking $\&$ Finance, 79:95-109.

Zhou, X. and Li, D. (2000). Continuous-time mean-variance portfolio selection: A stochastic lq framework. Applied Mathematics and Optimization, 42:19-33. 


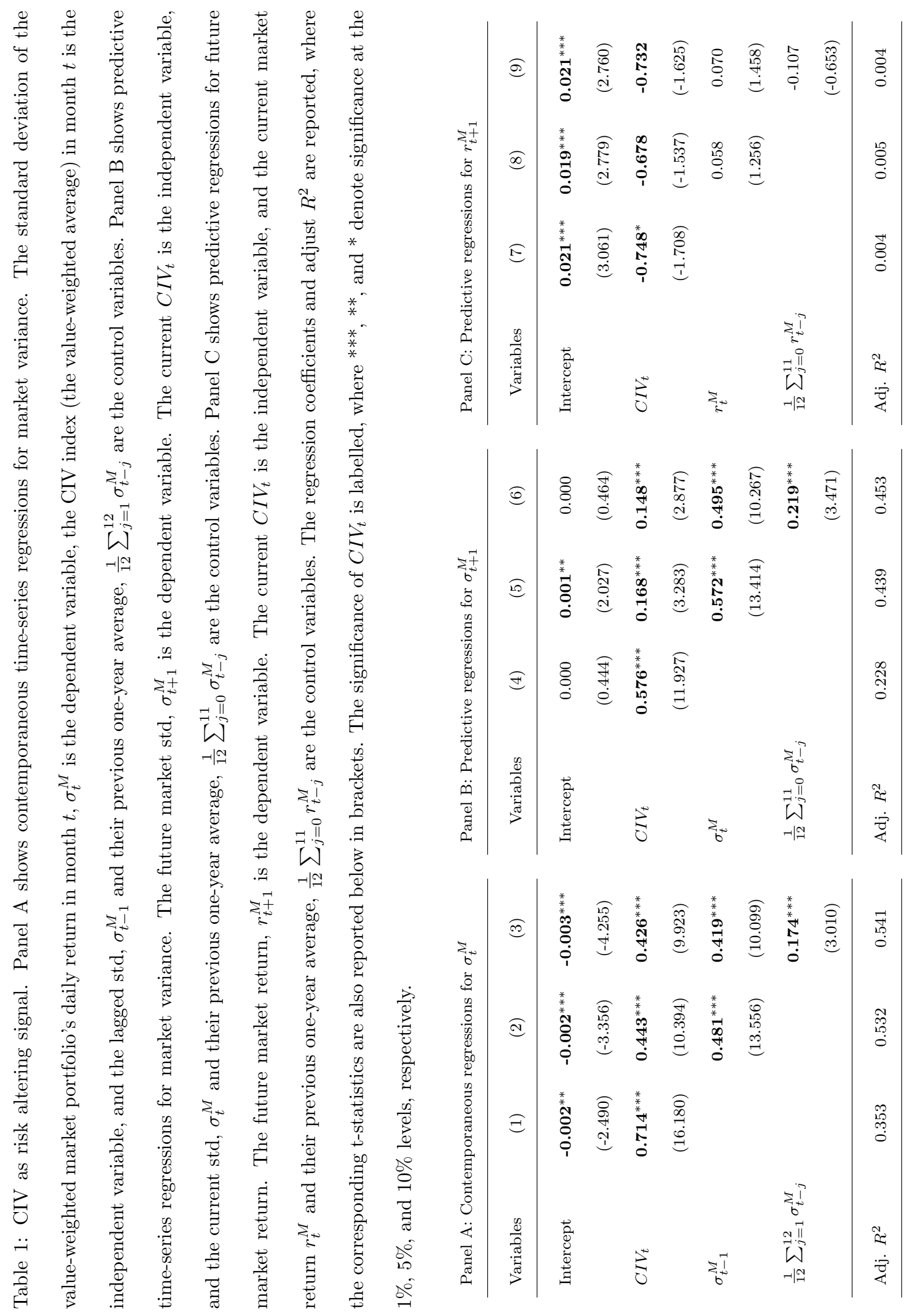


Table 2: The out-of-sample performances of three strategies for market timing (with transaction cost). The out-of-sample period is Jan. 1986 - Dec. 2019. The average holding position (position), average trading volume (trade) are the average values across all periods within a single multi-period investment practice and across all dynamic investment practices with the same length of investment horizon, which are scaled by $1 / 10^{9}$. The terminal wealth (wealth), cumulative risk penalty (risk penalty), cumulative transaction cost (TC), objective are the average values across all investment practices with the same length of investment horizon, which also be scaled by $1 / 10^{9}$. The Sharpe ratio (SR) and the net-of-cost Sharpe ratio (net-SR) are computed based on the (net-of-cost) terminal wealth levels of all investment practices with the same length of investment horizon. (net) SR gain ratios represent contribution percentages from timing expected return and from timing idiosyncratic volatility, which measured by switching from timing expected return strategy $x_{t}^{r}$ to our fully optimal strategy $x_{t}^{r \sigma}$.

Panel A: 12 months horizon (396 realizations)

\begin{tabular}{lccccccccc}
\hline strategy & position & trade & wealth & risk penalty & TC & objective & SR & net SR & SR gain ratio \\
\hline$x_{t}^{b}$ & 3.5846 & 0.4298 & 0.3804 & 0.0223 & 0.9724 & -0.6144 & 0.348 & -0.098 & \\
$x_{t}^{r}$ & 3.5800 & 0.4298 & 0.4083 & 0.0163 & 0.5003 & -0.1083 & 0.463 & -0.085 & $5 \%$ \\
$x_{t}^{r \sigma}$ & 3.4323 & 0.4112 & 0.4009 & 0.0112 & 0.2663 & 0.1235 & 0.506 & 0.178 & $95 \%$ \\
\hline
\end{tabular}

Panel B: 24 months horizon (384 realizations)

\begin{tabular}{lccccccccc}
\hline strategy & position & trade & wealth & risk penalty & TC & objective & SR & net SR & SR gain ratio \\
\hline$x_{t}^{b}$ & 12.1183 & 0.7390 & 2.7292 & 0.5450 & 5.4116 & -3.2274 & 0.428 & -0.101 & \\
$x_{t}^{r}$ & 12.2167 & 0.7455 & 2.9361 & 0.4268 & 3.2289 & -0.7196 & 0.551 & -0.055 & $11 \%$ \\
$x_{t}^{r \sigma}$ & 11.8835 & 0.7235 & 2.9735 & 0.2968 & 1.7189 & 0.9578 & 0.662 & 0.316 & $89 \%$ \\
\hline
\end{tabular}

Panel C: 36 months horizon (372 realizations)

\begin{tabular}{lccccccccc}
\hline strategy & position & trade & wealth & risk penalty & TC & objective & SR & net SR & SR gain ratio \\
\hline$x_{t}^{b}$ & 23.5968 & 0.9608 & 8.9767 & 3.1429 & 13.5975 & -7.7637 & 0.498 & -0.085 & \\
$x_{t}^{r}$ & 23.7125 & 0.9665 & 9.1379 & 2.4964 & 8.6746 & -2.0331 & 0.589 & 0.012 & $19 \%$ \\
$x_{t}^{r \sigma}$ & 23.8458 & 0.9735 & 10.1401 & 1.6642 & 4.8032 & 3.6727 & 0.770 & 0.434 & $81 \%$ \\
\hline
\end{tabular}


Table 3: The out-of-sample performances of three strategies for five industries investment. The out-ofsample period is from Jan. 1986 to Dec. 2019. The terminal wealth (wealth), cumulative risk penalty (risk penalty), cumulative transaction cost (TC), objective are the average values computed across all investment practices with the same length of investment periods, which are scaled by $1 / 10^{9}$. The Sharpe ratio (SR) and the net-of-cost Sharpe ratio (net-SR) are computed based on the (net-of-cost) terminal wealth levels of all investment practices with the same length of investment horizon. SR gain ratios represent contribution percentages from timing expected return and from timing idiosyncratic volatility.

\begin{tabular}{lccccccc} 
Panel A: 12 months horizon (396 realizations) & & & & \\
\hline strategy & wealth & risk penalty & TC & objective & SR & net SR & SR gain ratio \\
\hline $\boldsymbol{x}_{t}^{b}$ & 0.6677 & 0.0768 & 2.5146 & -1.9238 & 0.343 & -0.293 & \\
$\boldsymbol{x}_{t}^{r}$ & 0.7969 & 0.0401 & 1.1594 & -0.4025 & 0.528 & -0.163 & $36 \%$ \\
$\boldsymbol{x}_{t}^{r \sigma}$ & 0.9226 & 0.0342 & 0.8216 & 0.0668 & 0.631 & 0.064 & $64 \%$ \\
\hline Panel B: 24 months horizon (384 realizations) & & & & \\
\hline strategy & wealth & risk penalty & TC & objective & SR & net SR & SR gain ratio \\
\hline $\boldsymbol{x}_{t}^{b}$ & 4.8323 & 1.9157 & 15.7477 & -12.8311 & 0.423 & -0.312 & \\
$\boldsymbol{x}_{t}^{r}$ & 5.5326 & 1.0362 & 7.6226 & -3.1262 & 0.620 & -0.156 & $38 \%$ \\
$\boldsymbol{x}_{t}^{r \sigma}$ & 6.4376 & 0.9827 & 5.5418 & -0.0869 & 0.778 & 0.095 & $62 \%$ \\
\hline Panel C: 36 months horizon (372 realizations) & & & & \\
\hline strategy & wealth & risk penalty & TC & objective & SR & net SR & SR gain ratio \\
\hline $\boldsymbol{x}_{t}^{b}$ & 17.6103 & 10.6642 & 41.4635 & -34.5175 & 0.559 & -0.284 & \\
$\boldsymbol{x}_{t}^{r}$ & 17.6974 & 5.8122 & 20.2676 & -8.3824 & 0.697 & -0.068 & $45 \%$ \\
$\boldsymbol{x}_{t}^{r \sigma}$ & 21.3764 & 6.3946 & 15.9439 & -0.9620 & 0.881 & 0.195 & $55 \%$ \\
\hline
\end{tabular}


Figure 1: The holding positions and trading volumes of three different strategies from Jan. 1986 to Dec. 2019 (market timing without transaction cost). The strategies include baseline strategy, timing expected return strategy, timing idiosyncratic volatility strategy (our fully optimal strategy).

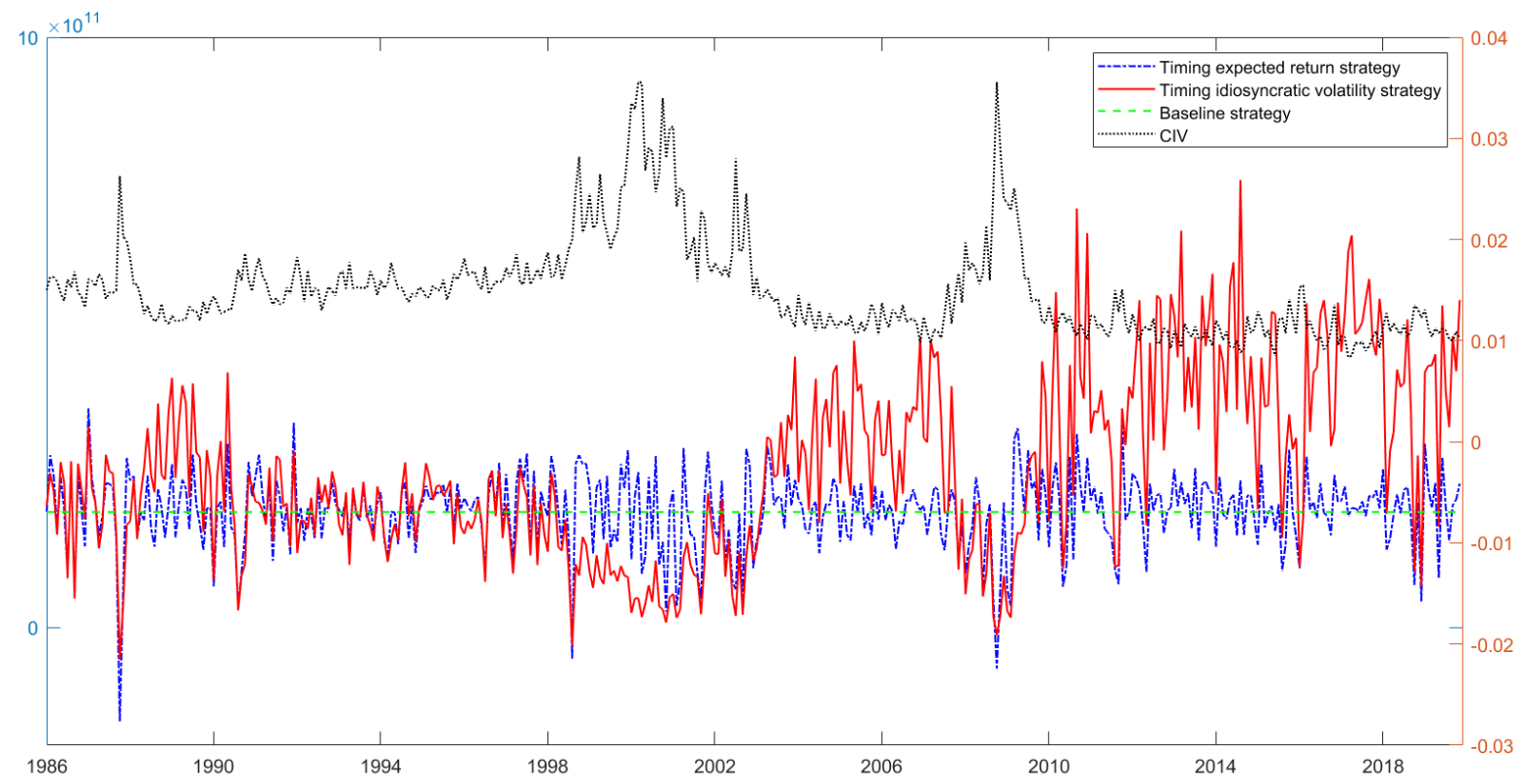

(a) Holding positions

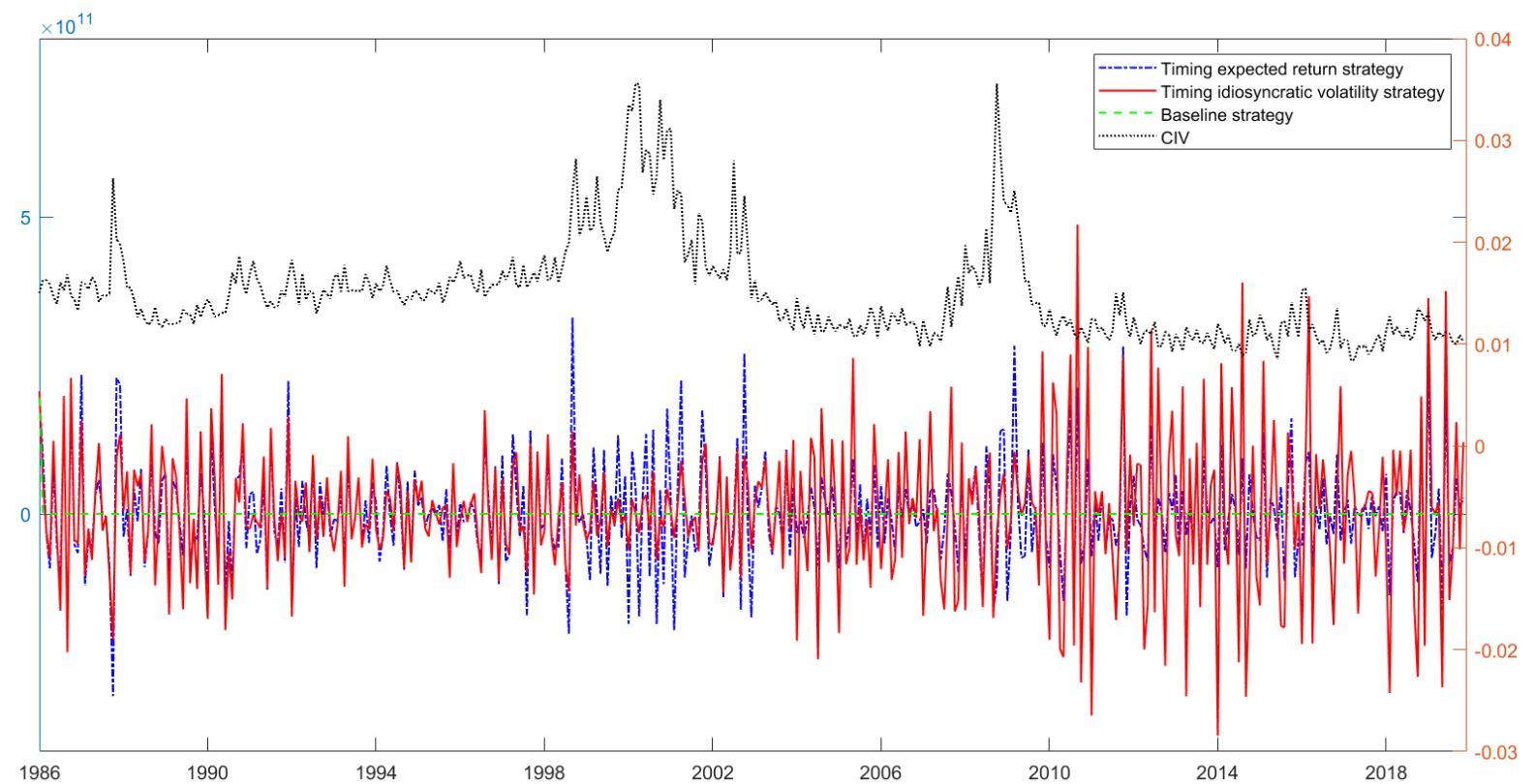

(b) Trading volumes 
Figure 2: The wealth, cumulative risk penalty, objective for three different strategies from Jan. 1986 to Dec. 2019 (market timing without transaction cost). The strategies include baseline strategy, timing expected return strategy, timing idiosyncratic volatility strategy (our fully optimal strategy). The cumulative risk penalty is computed based on the realized idiosyncratic volatility of the value-weighted market portfolio. The objective is equal to the wealth minus the cumulative risk penalty.

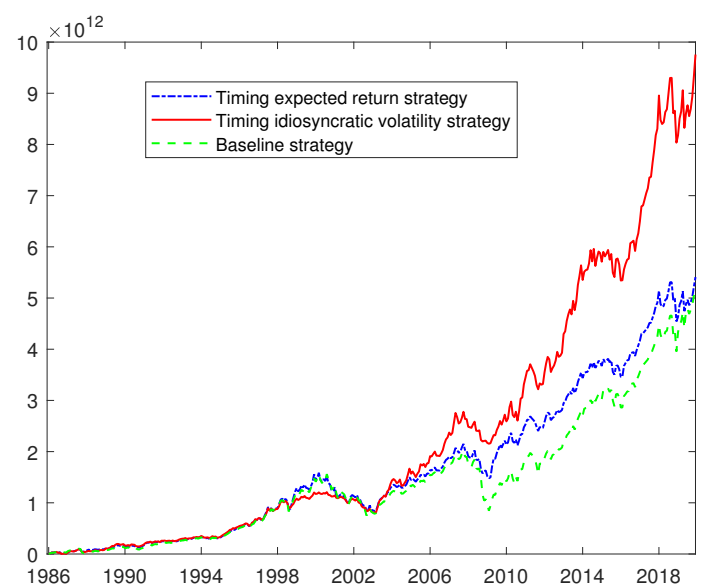

(a) Wealth

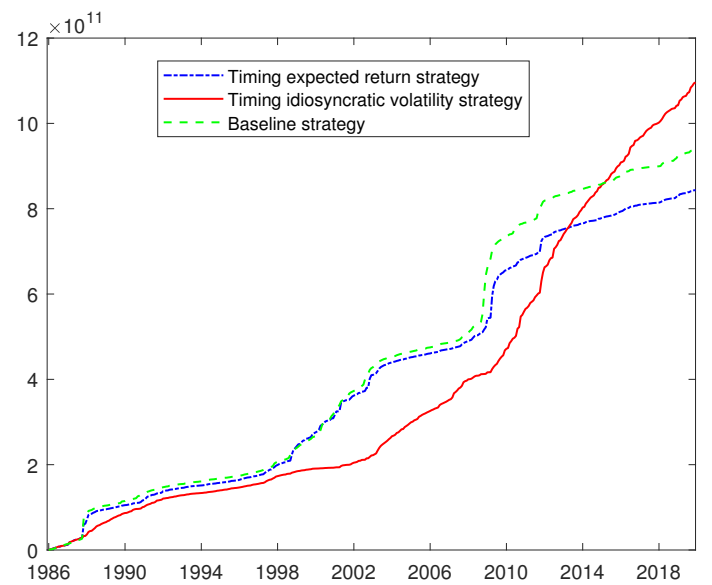

(b) Cumulative risk penalty

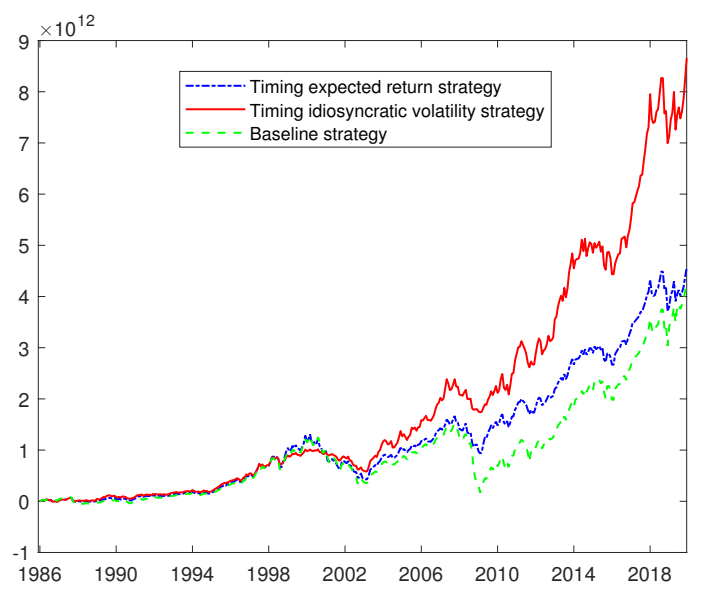

(c) Objective 
Figure 3: Zoom in 2008 crisis. The holding positions of the three strategies from Jan. 2008 to Jane. 2011 are plotted. The strategies include baseline strategy, timing expected return strategy, timing idiosyncratic volatility strategy (our fully optimal strategy).

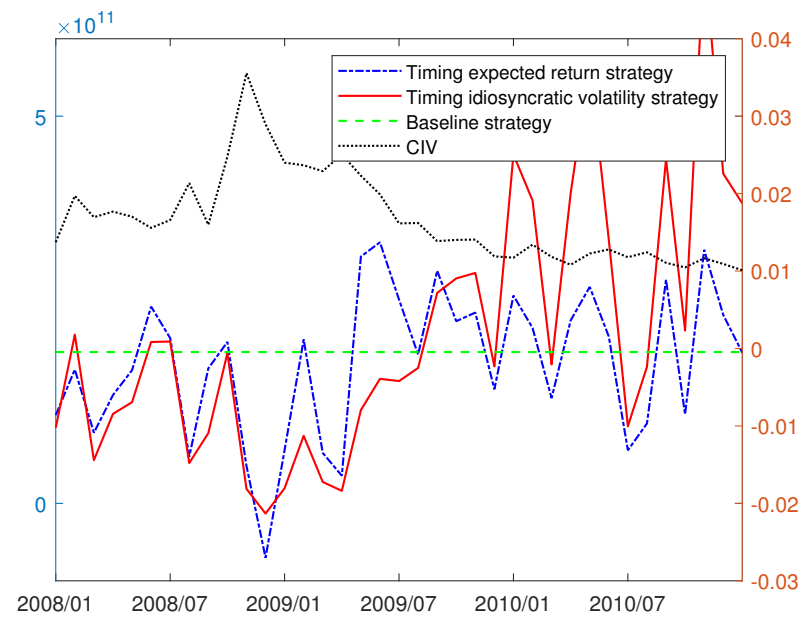


Figure 4: The benefits from timing expected return and timing idiosyncratic volatility (market timing case). The heights of blue pillars represent the increment/reduction ratio on wealth, risk penalty and transaction cost, by switching from baseline strategy (which does not do any timing) to timing expected return strategy. The heights of red pillars represent the additional benefits from timing idiosyncratic volatility, i.e., switching from timing expected return strategy to our fully optimal strategy (which conditions on expected returns as well as idiosyncratic volatility).

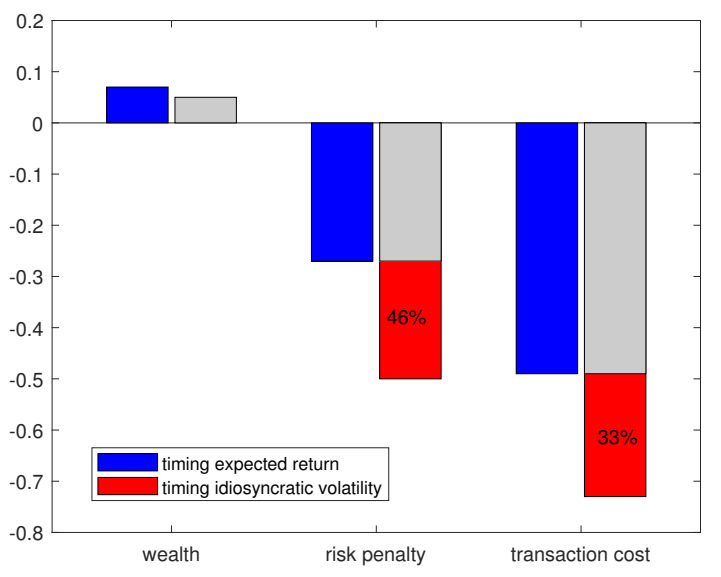

(a) 12 months

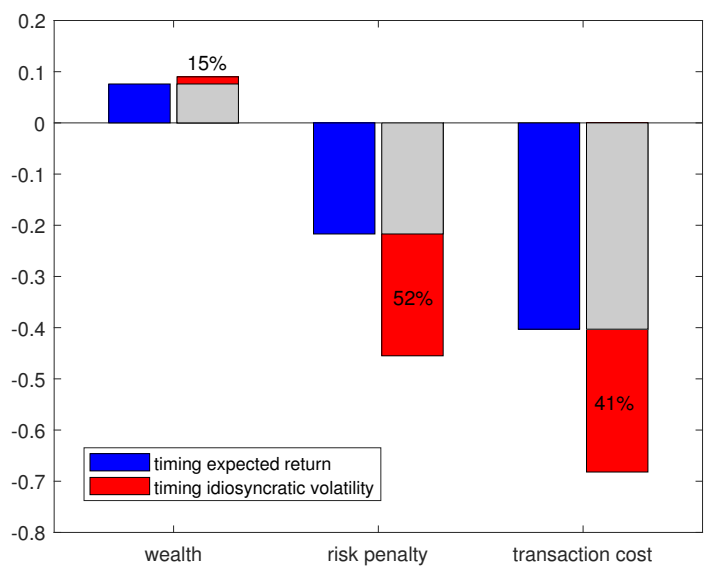

(b) 24 months

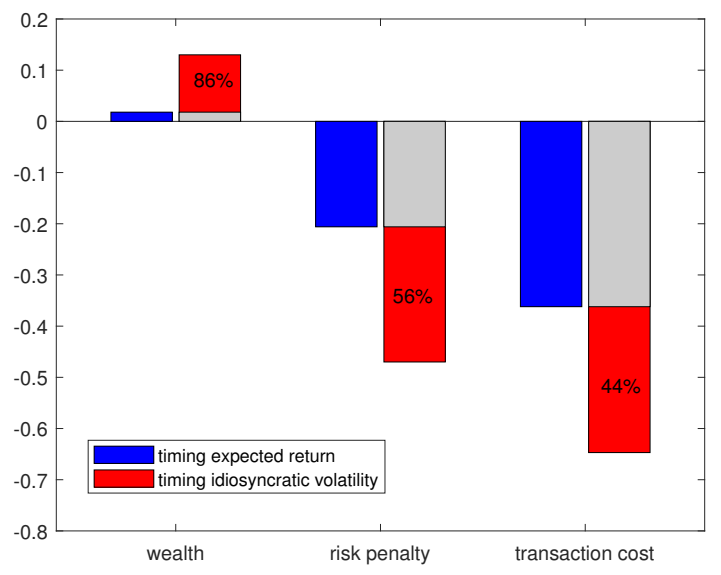

(c) 36 months 
Figure 5: The performance of a 24 months market timing from Jan. 2000 to Dec. 2001 with a high level of CIV (conservative type strategy).

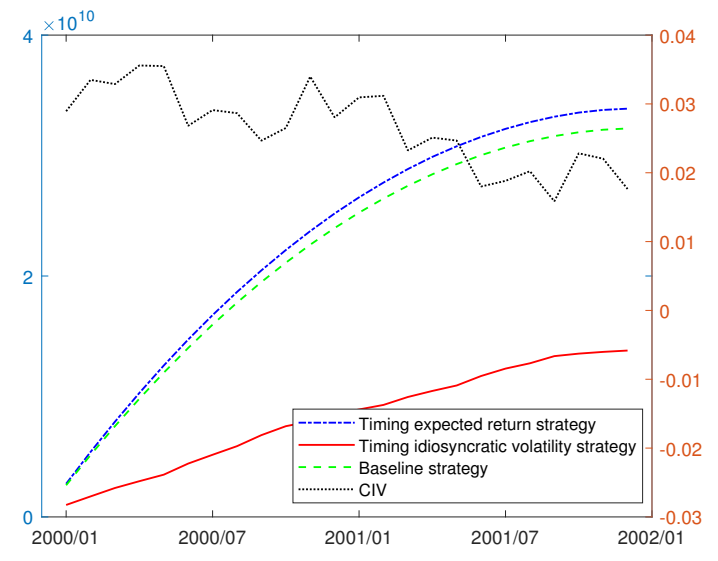

(a) Holding position

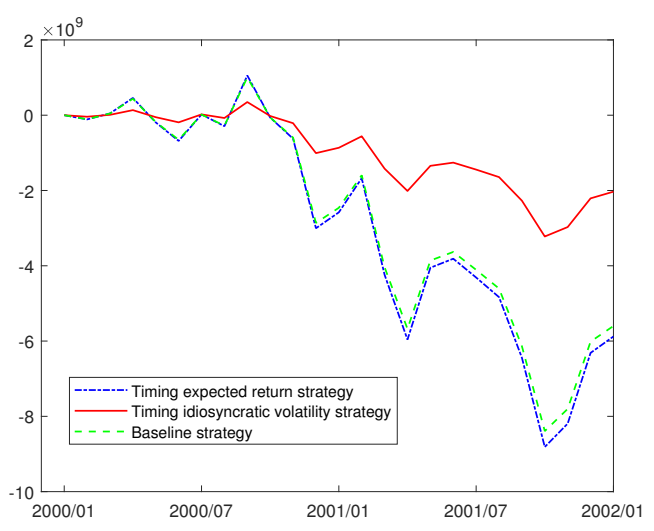

(c) Wealth

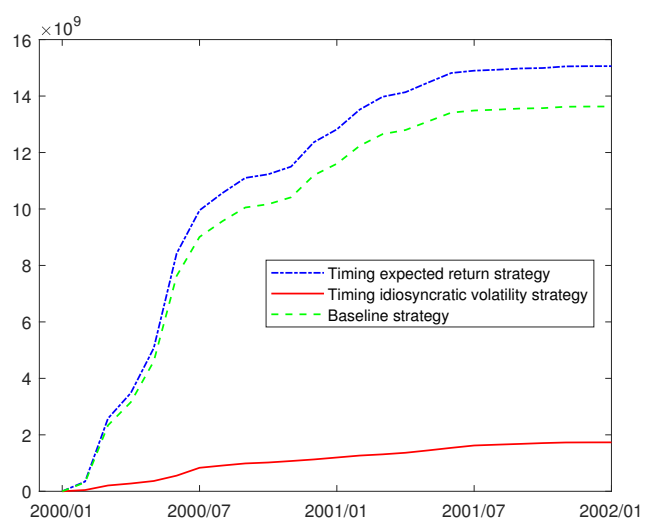

(e) Transaction cost

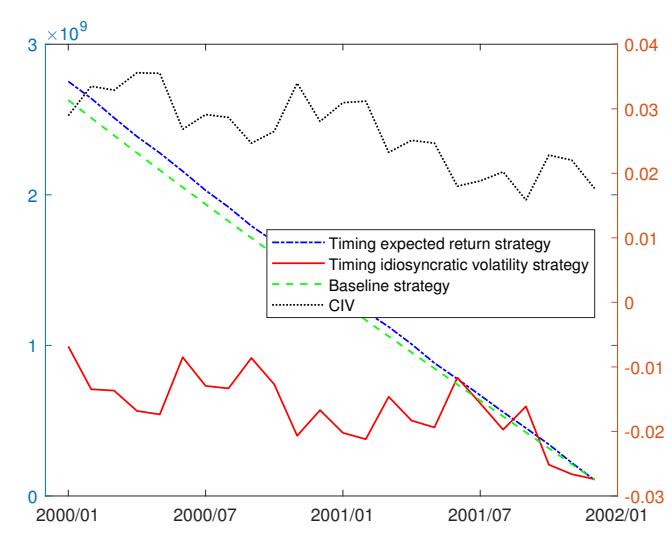

(b) Trading Volume

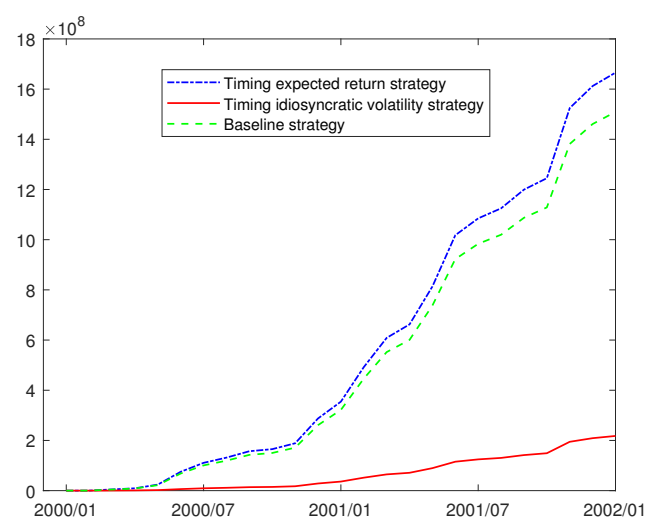

(d) Risk penalty

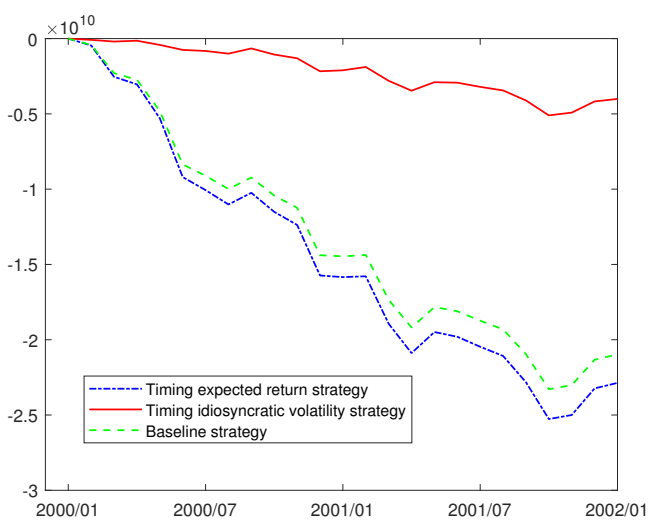

(f) Objective 
Figure 6: The performance of a 24 months market timing from Jan. 2003 to Dec. 2004 with a low level of CIV (aggressive type strategy).

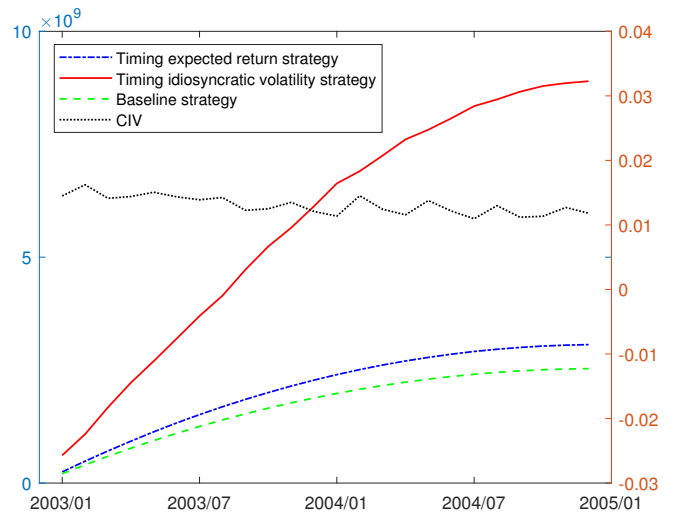

(a) Holding position

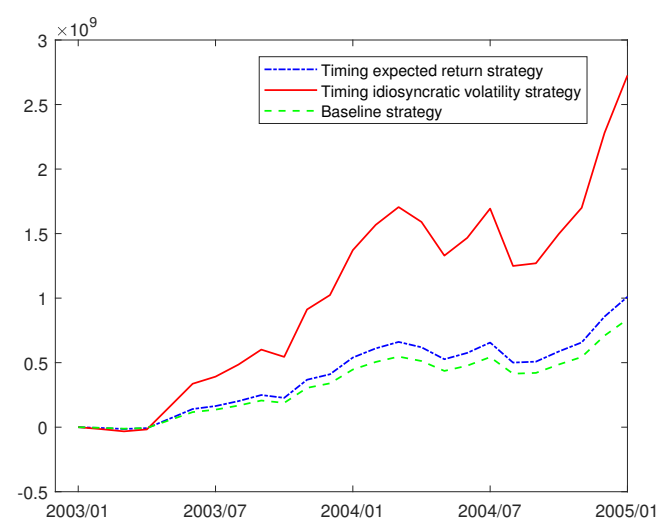

(c) Wealth

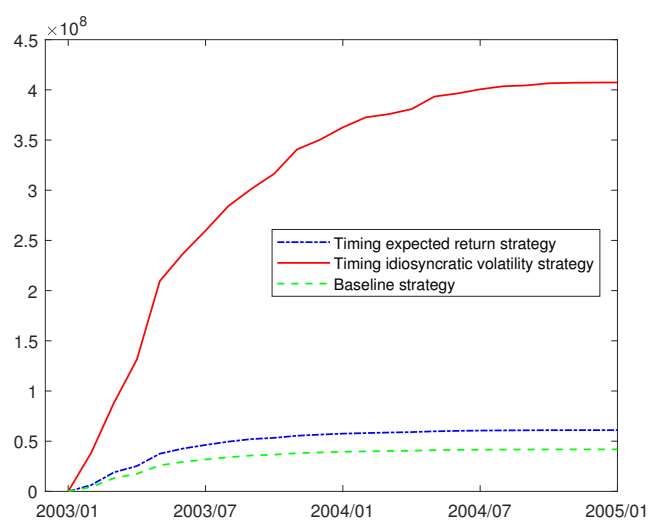

(e) Transaction cost

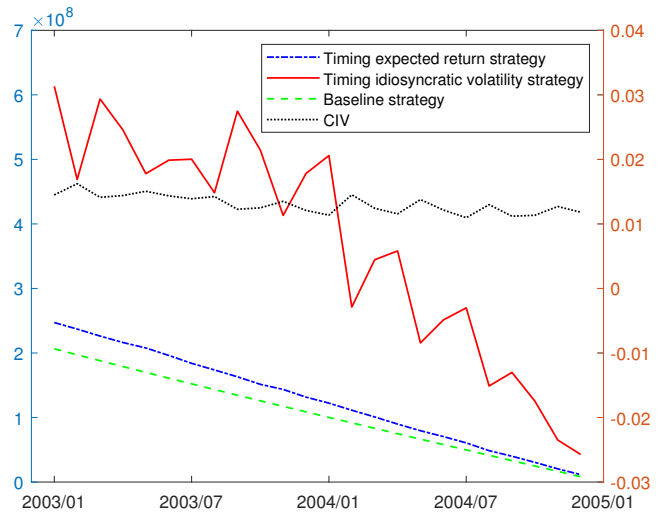

(b) Trading volume

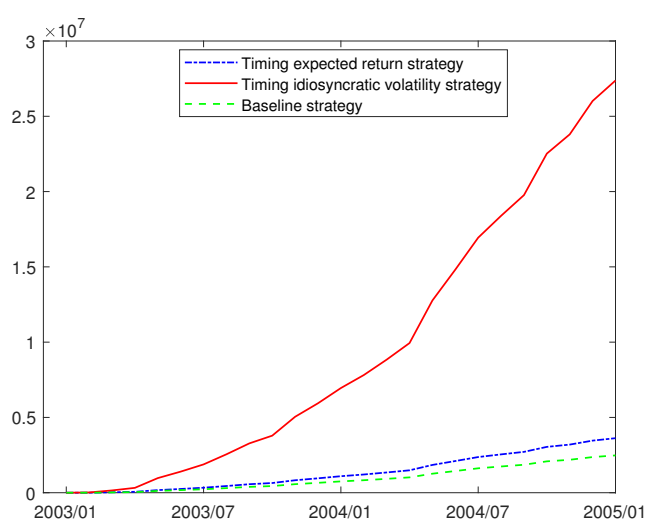

(d) Risk penalty

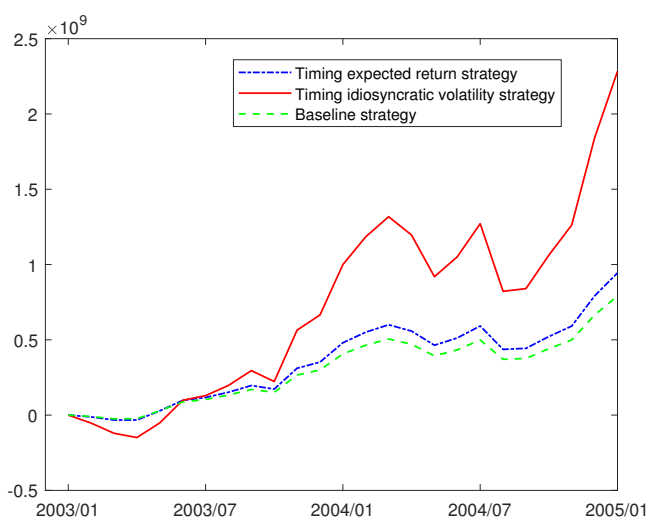

(f) Objective 
Figure 7: The empirical influences of parameter $\psi$ on the average trading rate. The analysis is based on the $12(24,36)$ months dynamic investment practices. The average trading rate during an investment is computed over the $12(24,36)$ investment periods. $\psi$ is the estimated persistence parameter in each dynamic investment.

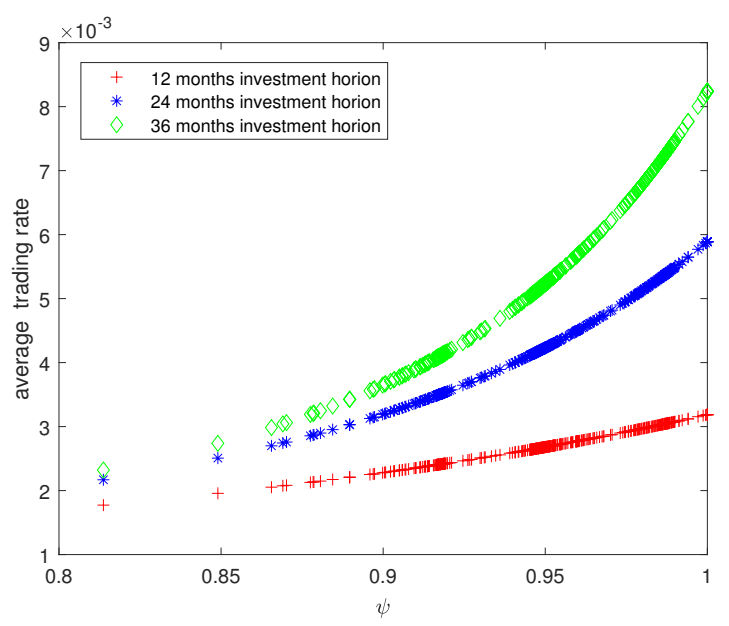


Figure 8: The increments of net-of-cost Sharpe Ratio achieved by timing expected return (blue bars) and timing idiosyncratic volatility (red bars). $N=1$ corresponds to market timing with transaction cost case, $N=5$ corresponds to five industry investment case. The height of pillars represent the absolute increments. The percentage number in the red bars represents relative contribution ratio from timing idiosyncratic volatility, measured by switching from timing expected return strategy to our fully optimal strategy (that is conditional on time-varying structure of both expected return and idiosyncratic volatility).

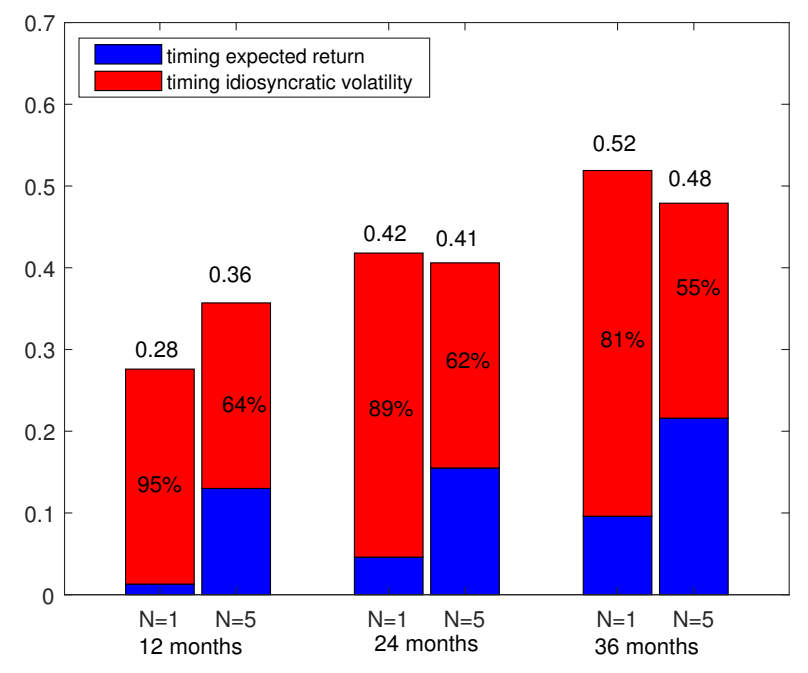

